\title{
Extended rotating disks around post-AGB stars ${ }^{\star} \star \star \star$
}

\author{
V. Bujarrabal ${ }^{1}$, J. Alcolea $^{2}$, H. Van Winckel ${ }^{3}$, M. Santander-García ${ }^{2,4}$, and A. Castro-Carrizo ${ }^{5}$ \\ ${ }^{1}$ Observatorio Astronómico Nacional (OAN-IGN), Apartado 112, 28803 Alcalá de Henares, Spain \\ e-mail: v.bujarrabal@oan.es \\ 2 Observatorio Astronómico Nacional (OAN-IGN), C/ Alfonso XII 3, 28014 Madrid, Spain \\ 3 Instituut voor Sterrenkunde, KU Leuven, Celestijnenlaan 200D, 2001 Leuven, Belgium \\ ${ }^{4}$ Centro de Astrobiología (CSIC-INTA), Ctra. M-108, km. 4, 28850 Torrejón de Ardoz, Madrid, Spain \\ 5 Institut de Radioastronomie Millimétrique, 300 rue de la Piscine, 38406 Saint Martin d'Hères, France
}

Received 4 June 2013 / Accepted 6 July 2013

\section{ABSTRACT}

\begin{abstract}
Context. There is a group of binary post-AGB stars that show conspicuous near-infrared (NIR) excess, which is usually assumed to arise from hot dust in very compact possibly rotating disks. These stars are surrounded by significantly fainter nebulae than the standard, well studied protoplanetary and planetary nebulae (PPNe, PNe).

Aims. We aim to identify and study extended rotating disks around these stars and shed light on the role of disks in the formation and shaping of planetary nebulae.

Methods. We present high-sensitivity mm-wave observations of CO lines in 24 objects of this type. The resulting CO lines are compared with profiles expected to arise from rotating disks from both theoretical and observational grounds. We derive simple formulae that allow us to determine the mass of the CO-emitting gas and estimate its extent. The reliability and uncertainty of the methods are also widely discussed.

Results. CO emission is detected in most observed sources, and the line profiles show that the emissions very probably come from disks in rotation. We derive typical values of the disk mass between $10^{-3}$ and $10^{-2} M_{\odot}$ about two orders of magnitude lower than the (total) masses of standard PPNe. The high-detection rate (upper limits being not very significant) clearly confirm that the NIR excess of these stars arises from compact disks in rotation, which are likely the inner parts of those found here. Low-velocity outflows are also found in about eight objects with moderate expansion velocities of $\sim 10 \mathrm{~km} \mathrm{~s}^{-1}$ to be compared with the velocities of about $100 \mathrm{~km} \mathrm{~s}^{-1}$ often found in standard PPNe. Except for two sources with complex profiles, the outflowing gas in our objects represents a minor nebular component. Our simple estimates of the typical disk sizes yields values $\sim 0.5-1$ arcsec, which is between $5 \times 10^{15}$ and $3 \times 10^{16} \mathrm{~cm}$. Estimates of the linear momenta carried by the outflows, which can only be performed in a few well studied objects, also yield moderate values when compared to the linear momenta that can be released by the stellar radiation pressure (contrary, again, to the case of the very massive and fast bipolar outflows in standard PPNe that are strongly overluminous). The mass and dynamics of nebulae around various classes of post-AGB stars differ very significantly, and we can expect the formation of PNe with very different properties.
\end{abstract}

Key words. stars: AGB and post-AGB - circumstellar matter - radio lines: stars - planetary nebulae: general

\section{Introduction: low- and high-mass nebulae around post-AGB stars}

Planetary and protoplanetary nebulae (PNe, PPNe) very often show axisymmetric shapes and fast axial movements, which are thought to be due to shock interaction between the very collimated post-AGB jets and the slow and isotropic asymptotic giant branch (AGB) wind (see Balick \& Frank 2002, and references therein). Many PPNe already contain most of the initial stellar mass (in some way, the star has become a nebula), and a significant fraction of this mass is accelerated by these shocks to values typically ranging from 50 to $200 \mathrm{~km} \mathrm{~s}^{-1}$ (see e.g. Bujarrabal et al. 2001). We note that the best studied objects tend to be relatively massive and luminous because of the usual observational bias. Most of the gas in most of these standard PPNe is in molecular form. However, the effects of photodissociation due to the hot

\footnotetext{
* Appendices are available in electronic form at http: //www . aanda.org

$\star \star$ Reduced spectra (FITS files) are available at the CDS via anonymous ftp to cdsarc.u-strasbg.fr (130.79.128.5) or via http://cdsarc.u-strasbg.fr/viz-bin/qcat?J/A+A/557/A104
}

central star are already noticeable in more evolved objects, and the gas is often composed of neutral or ionized atoms. In the final stage of planetary nebulae, many nebulae are found to contain in total of $\sim 0.2-1 M_{\odot}$ in the form of atomic or molecular gas (Huggins et al. 1996; Kimura et al. 2012; Castro-Carrizo et al. 2001; Fong et al. 2001).

In well studied (massive) nebulae, the formation of these axial structures involves enormous amounts of energy and linear momentum, well above what the stellar radiation can provide. On the other hand, the main phase of the PN shaping, when the shocks are actually accelerating the nebular material, is extremely short, lasting just a few hundred years (Bujarrabal et al. 2001; in total, the whole protoplanetary phase is thought to last $\sim 1000 \mathrm{yr}$, and the typical lifetime of the PN phase, when the nebula just follows a ballistic expansion, is $\sim 10000 \mathrm{yr}$ ).

The presence of gas disks orbiting post-AGB stars is often postulated to explain the very energetic PPN dynamics and the collimation of the post-AGB jets (see e.g. Soker 2002; Frank \& Blackman 2004). Mass reaccretion from rotating disks (in the presence of a magnetic field) can provide the required energy and momentum in a process similar to that at work in forming 
stars. In the case of evolved stars, the presence of a stellar or substellar companion is required to explain the extra angular momentum that is necessary to form a rotating disk from previously ejected material, since it is expelled with negligible angular momentum. The presence of rotating disks around post-AGB stars and their main properties is, therefore, a basic question to understand the post-AGB ejections and also the spectacular and very fast evolution of the shape and dynamics of young PNe.

We recall that torus-like or oblate structures of molecular gas are commonly detected in the central equatorial regions of protoplanetary and young planetary nebulae, although they are not observed to rotate but to be systematically in expansion (like the rest of the nebula). These expanding structures are usually thought to be mere remnants of the former AGB winds. See the cases of M 1-92 (Alcolea et al. 2007), M2-9 (Castro-Carrizo et al. 2012), M 2-56 (Castro-Carrizo et al. 2002), etc.

Not all post-AGB stars are surrounded by such thick shells. There is a population of faint (evolved) PNe in which only significantly lower amounts of mass $\left(<0.1 M_{\odot}\right)$ have been detected (Acker et al. 2012; Huggins et al. 1996; Pottasch 1984), possibly because most of the mass is placed in extended low-brightness haloes or simply due to the lack of nebular material. These objects are less studied than more massive PNe.

There is also a group of early post-AGB objects in which the stellar component dominates and that are surrounded by lowmass nebulae. These objects are old yellow low-gravity and relatively luminous stars (but in general less luminous than the massive standard PPNe presented in previous paragraphs). Most of them also show relatively low stellar temperatures at usually $<10000 \mathrm{~K}$. According to these properties, they are thought to have evolved from a previous red giant phase but to be still far from the blue or white dwarf stage (see e.g. van Winckel 2003). Contrary to the case of massive protoplanetary nebulae, most of these stars are bright in the optical, since the circumstellar extinction is not high, so they are often identified from their stellar properties.

All these optically-bright post-AGB objects show a clear farinfrared (FIR) excess, since some nebular material is present in any case. A significant fraction of them also show a remarkable near-infrared (NIR) excess, which has been attributed to the emission of hot dust at about $1000 \mathrm{~K}$. The high temperature would show that dust grains are kept close to the star, in spite of the expected low mass-loss rate after the end of the AGB and the slow evolution of these sources. A systematic search for bright post-AGB stars (see van Winckel et al. 2009; van Aarle et al. 2012, and references therein) resulted in catalogs in which about half of the candidates show a NIR excess of this kind; among these are the RV Tauri pulsators. Often these objects show a peculiar photospheric composition which is depleted in refractory elements. This is likely due to reaccretion of only the gas from the dusty circumstellar environment (Waters et al. 1992). Another observational characteristic of these objects is that the infrared dust spectra indicate relatively large grains with a very high crystallinity fraction (Gielen et al. 2011). All these observational characteristics suggest that these stars are surrounded by very compact and stable nebular components, which are possibly rotating disks, see Van Winckel (2003). The compact nature of the NIR emitting component is confirmed by interferometric experiments (Deroo et al. 2007), which also suggested an elongated, disk-like shape. Practically all the stars of this kind (bright post-AGB stars with NIR excess) that have been studied in detail are close binaries (e.g. Van Winckel et al. 1999; de Ruyter et al. 2005), which would explain the origin of the angular momentum required to form the disks. The exceptions could still be objects in which the study of the radial velocity variations is hampered by the stellar pulsation.

However, the rotation dynamics of these small disks is not yet well established, because no spectroscopic data have been able to probe their velocity field. We cannot discard that the presence of hot dust is due to a very recent mass ejection or expansion at a very low velocity. Indeed, very slow outflowing gas was found in the very inner regions of some standard PPNe, probably ejected during the very last AGB phases (Sánchez Contreras et al. 2004; Castro-Carrizo et al. 2012).

Among these objects with significant NIR excess, we can mention the variable stars of RV Tau type, the Red Rectangle, 89 Her, HR 4049, etc. (see e.g. Trams et al. 1991; Alcolea \& Bujarrabal 1991; Waters et al. 1992; Van Winckel 2003). In the Red Rectangle and 89 Her, which are probably the best studied of these sources, the total nebular mass is $\lesssim 0.01 M_{\odot}$ (e.g. Bujarrabal et al. 2001, 2005, 2007). The other objects, in which the studies of the nebular component are less detailed, show a nebular mass that is always lower than $0.1 M_{\odot}$, as seen in Alcolea \& Bujarrabal (1991), Van Winckel (2003), etc. We cannot exclude the presence of outer haloes or very inner components with significant amounts of mass (Men'shchikov et al. 2002), but these are difficult to detect.

Only in one object, the Red Rectangle, has the presence of an extended disk in rotation been demonstrated by means of interferometric maps of mm-wave CO lines (Bujarrabal et al. 2005). On the other hand, the detailed analysis of Herschel observations of FIR high- $J$ lines by Bujarrabal \& Alcolea (2013) suggested that the presence of diffuse gas expanding at about $10 \mathrm{~km} \mathrm{~s}^{-1}$ (probably in the form of axial blobs) is necessary to explain the relatively intense line-wing emission in this object.

89 Her was also mapped in low- $J$ CO emission (Bujarrabal et al. 2007). The nebula is dominated by an hour-glass like structure about $8^{\prime \prime}$ wide and expanding at about $5 \mathrm{~km} \mathrm{~s}^{-1}$. There is also a central intense component, practically unresolved in the observations $\left(\lesssim 1^{\prime \prime}\right)$, which was suggested to be in rotation in view of its low velocities and extent.

Other previously published $\mathrm{CO}$ observations of these postAGB stars yielded less detailed results (see Alcolea \& Bujarrabal 1991; Bujarrabal et al. 1988, 1990; Maas et al. 2003), and only two more objects were detected. R Sct (a peculiar RV Tau variable) shows a narrow but complex single-dish profile. IRAS 08544-4431 showed relatively narrow profiles with a prominent central peak. AC Her (another RV Tau star) may show a narrow ${ }^{12} \mathrm{CO} J=2-1$ line, but it is weak and was just tentatively detected. Other similar objects were observed but not detected in those observations.

Is the extended rotating disk of the Red Rectangle a very peculiar characteristic of this nebula? Or are rotating disks, on the contrary, present in many other post-AGB objects? Are rotating equatorial disks and expanding axial blobs simultaneously present (at least in low-mass post-AGB nebulae)? Can we invoke disks to explain the dynamics of massive nebulae, though we do not find any observational sign of the presence of rotating structures in them? This paper is devoted to shed some light onto these questions.

\section{Source sample}

We have selected 24 sources to be observed in $\mathrm{CO}$ mm-wave lines following three criteria:

1. They have been identified as post-AGB stars, in view of their evolved nature (not associated with interstellar clouds and 
Table 1. Post-AGB sources observed with the $30 \mathrm{~m}$ IRAM telescope.

\begin{tabular}{|c|c|c|c|c|c|c|c|c|c|}
\hline Source & \multicolumn{2}{|c|}{$\begin{array}{c}\text { Observed coordinates } \\
\mathrm{J} 2000 \\
\end{array}$} & $\begin{array}{c}F_{25 \mu} \\
\mathrm{Jy}\end{array}$ & $\begin{array}{c}F_{60 \mu} \\
\mathrm{Jy}\end{array}$ & $\begin{array}{c}\text { Luminosity } \\
L_{\odot} \\
\end{array}$ & $\begin{array}{c}\text { Distance } \\
\mathrm{pc}\end{array}$ & sp. type & $\begin{array}{c}V_{*}(\mathrm{LSR}) \\
\mathrm{km} \mathrm{s}^{-1} \\
\end{array}$ & Comments \\
\hline RV Tau & $04: 47: 06.73$ & $+26: 10: 45.6$ & 18.1 & 6.5 & 3500 & $2200^{1}$ & G2-M2 & +17 & RV Tau variable \\
\hline DY Ori & $06: 06: 14.91$ & $+13: 54: 19.1$ & 14.9 & 4.2 & 1600 & $2000^{1}$ & G0 I & -16 & RV Tau variable \\
\hline Red Rectangle & $06: 19: 58.22$ & $-10: 38: 14.7$ & 456.1 & 173.1 & 6000 & $710^{2}$ & B9 & 0 & interferometric $\mathrm{CO}$ maps \\
\hline U Mon & $07: 30: 47.47$ & $-09: 46: 36.8$ & 88.4 & 26.6 & 4000 & $800^{1}$ & F8-K0 I & +9 & RV Tau variable \\
\hline $\mathrm{AI} \mathrm{CMi}$ & $07: 35: 41.15$ & $+00: 14: 58.0$ & 68.1 & 18.5 & $3000^{3}$ & $1500^{3}$ & G5 I & +33 & \\
\hline HR 4049 & $10: 18: 07.59$ & $-28: 59: 31.2$ & 9.6 & 1.8 & 2900 & $650^{4}$ & A6 I & -45 & well studied inner disk \\
\hline $89 \mathrm{Her}$ & $17: 55: 25.19$ & $+26: 03: 00.0$ & 54.5 & 13.4 & 9200 & $1000^{5}$ & F2 I & -8 & interferometric CO maps \\
\hline IRAS $18123+0511$ & $18: 14: 49.39$ & $+05: 12: 55.7$ & 11.0 & 4.2 & $3000^{3}$ & $3500^{3}$ & $\mathrm{~F} 2$ & +99 & \\
\hline AC Her & $18: 30: 16.24$ & $+21: 52: 00.6$ & 65.3 & 21.4 & 3500 & $1100^{1}$ & F2-K4 I & -10 & previous tent. det., RV Tau variable \\
\hline R Sct & $18: 47: 28.95$ & $-05: 42: 18.5$ & 9.3 & 8.2 & 14000 & $1000^{5}$ & G0-K0 I & +55 & previous det., pec. RV Tau variable \\
\hline IRAS $19125+0343$ & 19:15:01.18 & $+03: 48: 42.7$ & 26.5 & 7.8 & $3000^{3}$ & $1500^{3}$ & $\mathrm{~F} 2$ & +84 & \\
\hline IRAS 19157-0247 & $19: 18: 22.71$ & $-02: 42: 10.9$ & 7.2 & 2.5 & $3000^{3}$ & $2900^{3}$ & F3 & +49 & \\
\hline IRAS $20056+1834$ & 20:07:54.62 & $+18: 42: 54.5$ & 18.0 & 5.4 & $3000^{3}$ & $3000^{3}$ & G0 & $\sim 0$ & edge-on disk \\
\hline R Sge & 20:14:03.75 & $+16: 43: 35.1$ & 7.5 & 2.1 & 2500 & $2500^{1}$ & G0-K0 I & +28 & RV Tau variable \\
\hline
\end{tabular}

Notes. Observed coordinates and other relevant properties including IRAS fluxes, total luminosity and adopted distance (Sect. 2.1), stellar spectral type, and stellar velocity (derived from optical lines, except for sources previously detected in CO emission) are listed. Distance estimate: ${ }^{(1)} \mathrm{P}-\mathrm{L}$ relation for RV Tau variables; ${ }^{(2)}$ Men'shchikov et al. (2002); ${ }^{(3)}$ Assumed luminosity; ${ }^{(4)}$ Corrected parallax (Acke et al. 2013); ${ }^{(5)}$ Hipparcos parallax.

Table 2. Southern post-AGB sources observed with the APEX telescope.

\begin{tabular}{|c|c|c|c|c|c|c|c|c|c|}
\hline \multirow{2}{*}{$\begin{array}{l}\text { Source } \\
\text { AR Pup }\end{array}$} & \multicolumn{2}{|c|}{$\begin{array}{c}\text { Observed coordinates } \\
\mathrm{J} 2000 \\
\end{array}$} & \multirow{2}{*}{$\begin{array}{c}F_{25 \mu} \\
\mathrm{Jy}\end{array}$} & \multirow{2}{*}{$\begin{array}{l}F_{60 \mu} \mu \\
\mathrm{Jy}\end{array}$} & \multirow{2}{*}{$\begin{array}{c}\text { Luminosity } \\
L_{\odot} \\
3500\end{array}$} & \multirow{2}{*}{$\begin{array}{c}\begin{array}{c}\text { Distance } \\
\mathrm{pc}\end{array} \\
2800^{1}\end{array}$} & \multirow{2}{*}{$\begin{array}{c}\text { sp. type } \\
\text { F0I }\end{array}$} & \multirow{2}{*}{$\begin{array}{c}\begin{array}{c}V_{*}(\mathrm{LSR}) \\
\mathrm{km} \mathrm{s}^{-1}\end{array} \\
+29\end{array}$} & \multirow{2}{*}{$\begin{array}{l}\text { Comments } \\
\text { RV Tau variable }\end{array}$} \\
\hline & 08:03:01.65 & $-36: 35: 47.9$ & & & & & & & \\
\hline IRAS 08544-4431 & $08: 56: 14.18$ & $-44: 43: 10.7$ & 158.8 & 56.3 & $3000^{2}$ & $550^{2}$ & F3 & +45 & previous det., RV Tau variable \\
\hline IW Car & $09: 26: 53.30$ & $-63: 37: 48.9$ & 96.2 & 34.5 & 3000 & $1000^{1}$ & A4I & -25 & RV Tau variable \\
\hline IRAS $10174-5704$ & $10: 19: 16.89$ & $-57: 19: 26.0$ & 60.3 & 16.1 & $3000^{2}$ & $850^{2}$ & G8I & +3 & \\
\hline IRAS $10456-5712$ & $10: 47: 38.40$ & $-57: 28: 02.7$ & 115.5 & 30.9 & $3000^{2}$ & $600^{2}$ & M1 & -9 & \\
\hline HD 95767 & $11: 02: 04.31$ & $-62: 09: 42.8$ & 15.7 & 10.9 & $3000^{2}$ & $1400^{2}$ & F3II & -32 & \\
\hline RU Cen & $12: 09: 23.81$ & $-45: 25: 34.8$ & 11.0 & 5.7 & 2500 & $2200^{1}$ & $\mathrm{G} 2$ & -35 & RV Tau variable \\
\hline HD 108015 & $12: 24: 53.50$ & $-47: 09: 07.5$ & 33.23 & 8.0 & $3000^{2}$ & $1800^{2}$ & F4I & -2 & \\
\hline IRAS 15469-5311 & $15: 50: 43.80$ & $-53: 20: 43.3$ & 42.1 & 15.5 & $3000^{2}$ & $1200^{2}$ & F3 & -14 & \\
\hline IRAS $15556-5444$ & $15: 59: 32.57$ & $-54: 53: 20.4$ & 17.8 & 7.1 & $3000^{2}$ & $1700^{2}$ & F8 & $?$ & \\
\hline
\end{tabular}

Notes. Observed coordinates and other properties are listed (see Table 1). Distance estimate: (1) P-L relation for RV tau stars. (2) Assumed luminosity.

the galactic plane), low gravity, relatively high luminosity, intermediate spectral type, and FIR excess (indicative of material ejected by the star). We selected sources from the catalog by de Ruyter et al. (2006); we also included low-mass post-AGB stars observed by Alcolea \& Bujarrabal (1991).

2. They all show NIR excess indicative of the presence of hot dust, possibly placed close to the star (Sect. 1). The NIR excess is very small in the case of $\mathrm{R}$ Sct, and particularly strong in IRAS $20056+1834$. In disk sources the viewing angle of the disk is an important parameter, which determines the spectral energy distribution (SED). For IRAS $20056+1834$, there is observational evidence that we look edge-on (Kameswara Rao et al. 2002), and the stellar light is severely obscured.

3. We selected intense sources in the FIR to increase the CO detection rate. The intense FIR would show that there is more extended material, in which we could find molecular gas, and/or the distance is not very large. A CO $-60 \mu \mathrm{m}$ flux correlation is found in standard (massive) PPNe (e.g. Bujarrabal et al. 1992), and there is also a hint of CO/FIR relation in the few previous observations of similar objects (Alcolea \& Bujarrabal 1991).
4. A first group of 14 sources was selected imposing that the declinations cannot be very negative $\left(\right.$ over $-25^{\circ}$ ) to be observed with the IRAM $30 \mathrm{~m}$ telescope. A second group of 10 southern sources was independently selected, to be observed with the APEX telescope.

The sources observed with the two telescopes, the IRAM $30 \mathrm{~m}$ telescope and the APEX telescope, are treated differently in this paper, because the data obtained with APEX are more difficult to interpret. The APEX data do not include ${ }^{13} \mathrm{CO} J=1-0$ lines, which are the basic data in our analysis and deduction of the main disk properties. Moreover, a significant fraction of the APEX observations suffer strong contamination due to galactic interstellar medium (ISM) emission, yielding poor limits to the disk emission. For these reasons, APEX data are more useful to complement the list of post-AGB objects showing disk emission than to estimate the detection rate and disk parameters.

The objects observed with the $30 \mathrm{~m}$ and APEX telescopes are respectively presented in Tables 1 and 2, where we give the observed coordinates, the IRAS 25 and $60 \mu$ m fluxes, luminosities, distances, stellar spectral types and velocities, and some comments. We have tried to derive the velocities of the stellar system center of gravity by averaging values from, if possible, 
many different observations; the uncertainty of the velocity determinations varies from object to object with a typical value of $\sim 5 \mathrm{~km} \mathrm{~s}^{-1}$.

\subsection{Estimates of the distance and luminosity of the studied objects}

Distances of the galactic disk sources are poorly constrained, since the sources are generally too far for direct distance detection via the parallax measurement. One of the exceptions is HR 4049 (Acke et al. 2013) for which the parallax estimate had to be corrected for the orbital motion of the primary around the center of mass. We adopted different strategies to estimate the distances of the other sources. For the RV Tauri stars we used the P-L relation of Alcock et al. (1998) calibrated to the Large Magellanic Cloud (LMC). We used the detailed study of the LMC RV Tauri pulsators of Gielen et al. (2009) to obtain better estimates of the intrinsic luminosities. We then modeled the SED similar to what has been performed in Gielen et al. (2011), using appropriate Kurucz model atmospheres and broadband photometric observations obtained using Simbad. We dereddened the raw photometric data to obtain the total line-of-sight reddening by $\chi^{2}$ minimization between the dereddened photospheric fluxes and the atmosphere model. The integration of the dereddened photospheric emission and the IR luminosity was then used to obtain the distances.

For nonpulsating objects for which there is no indication on the distance, we assumed a default luminosity of $3000 L_{\odot}$, modeled the SED, and determined the distance accordingly. IRAS 20056+1834 (Kameswara Rao et al. 2002; Gielen et al. 2011) is mainly an IR source, and the optical dereddened luminosity is dwarfed by the total infrared excess. The likely reason is that we look at the system edge-on and that the optical photons are dominated by scattered light. We therefore used the full SED integral as a proxy for the total luminosity and used this to constrain the distance. Another example in which we look at the system nearly edge-on is the Red Rectangle, for which the disk is resolved in the optical (Cohen et al. 2004); for the Red Rectangle we took the distance estimated by Men'shchikov et al. (2002) from their detailed modeling of the nebula emission. $\mathrm{R}$ Sct is a peculiar RV Tauri pulsator whose true nature is still a matter of debate (e.g. Matsuura et al. 2002). For this object we took the parallax measurement which yields a distance of $1 \mathrm{kpc}$ and obtained the luminosity by integrating the SED.

\section{Observations}

\subsection{IRAM $30 \mathrm{~m}$ data}

Data for the ${ }^{12} \mathrm{CO}$ and ${ }^{13} \mathrm{CO} J=2-1$ and $J=1-0$ lines have been taken with the IRAM $30 \mathrm{~m}$ telescope. The observations were performed in three observing sessions in May 2012, January 2013, and May 2013. We used the new EMIR (Eight MIxer) receivers, which are able to observe simultaneously in the $3 \mathrm{~mm}$ and $1 \mathrm{~mm}$ bands in dual polarization mode. During the first session, we observed the four CO lines simultaneously, but the two polarizations could be recorded only for the ${ }^{12} \mathrm{CO} J=2-1$ line. In the second and third sessions, we also observed the three weakest lines, ${ }^{12} \mathrm{CO} J=1-0$ and ${ }^{13} \mathrm{CO} J=2-1$ and $J=1-0$, in dual polarization. For each line, observations in the two polarizations were averaged after checking that the line intensities were compatible. The data were recorded using the FTS with a resolution of $200 \mathrm{kHz}$, which is equivalent

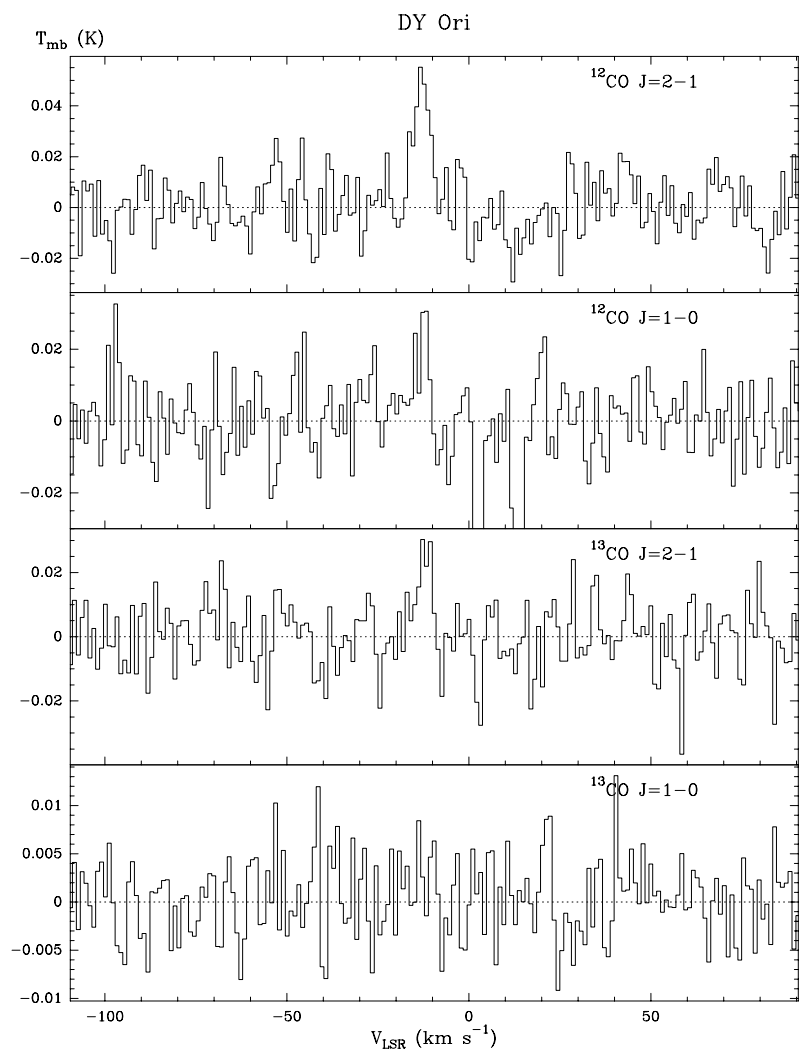

Fig. 1. Observed profiles in DY Ori.

to about 0.25 and $0.5 \mathrm{~km} \mathrm{~s}^{-1}$ in the $1 \mathrm{~mm}$ and $3 \mathrm{~mm}$ bands, respectively. In some cases, namely for very weak and/or wide lines, the spectral resolution was degraded by a factor 2 or 4 by averaging adjacent channels to better determine the line parameters.

The spatial resolution of the observations is $22-23^{\prime \prime}$ at $3 \mathrm{~mm}$ and $12-13^{\prime \prime}$ at $1 \mathrm{~mm}$ wavelength. Frequent pointing measurements were performed to monitor and correct pointing errors; errors not larger than $3^{\prime \prime}$ were typically found, which practically have no effects on the calibration. The observations were done by wobbling the subreflector by $2^{\prime}$ at a $0.5 \mathrm{~Hz}$ rate. This method is known to provide very stable and flat spectral baselines. The atmospheric conditions during the observations were good. The average zenith opacity during the observing runs was $\lesssim 0.3$, being slightly better at $110 \mathrm{GHz}$ and slightly worse at $115 \mathrm{GHz}$.

The data presented here have been calibrated in units of (Rayleigh-Jeans-equivalent) main-beam temperature corrected for the atmospheric attenuation, $T_{\mathrm{mb}}$, using the standard chopper wheel method. Calibration scans (observation of the hot and cold loads and of the blank sky) have been performed typically every 15-20 min. The temperature scale is set by observing hot and cold loads at ambient and liquid nitrogen temperatures. Correction for the antenna coupling to the sky and other losses have been done using the latest values for these parameters measured at the telescope. The sky attenuation is computed from the values of a weather station, the measurement of the sky emissivity, and a numerical model for the atmosphere at Pico de Veleta.

The observed $T_{\mathrm{mb}}$ values have been re-scaled after the observation of the well known emitters IRC +10216 (CW Leo) and NGC 7027, whose CO emission is strong and well characterized. For IRC +10216 , we adopted $T_{\mathrm{mb}}$ values at the line centers of $50 \mathrm{~K}, 36 \mathrm{~K}, 23 \mathrm{~K}$, and $1 \mathrm{~K}$, for respectively the ${ }^{12} \mathrm{CO}$ and ${ }^{13} \mathrm{CO} J=2-1$ and the ${ }^{12} \mathrm{CO}$ and ${ }^{13} \mathrm{CO} J=1-0$ transitions. 
V. Bujarrabal et al.: Extended rotating disks around post-AGB stars

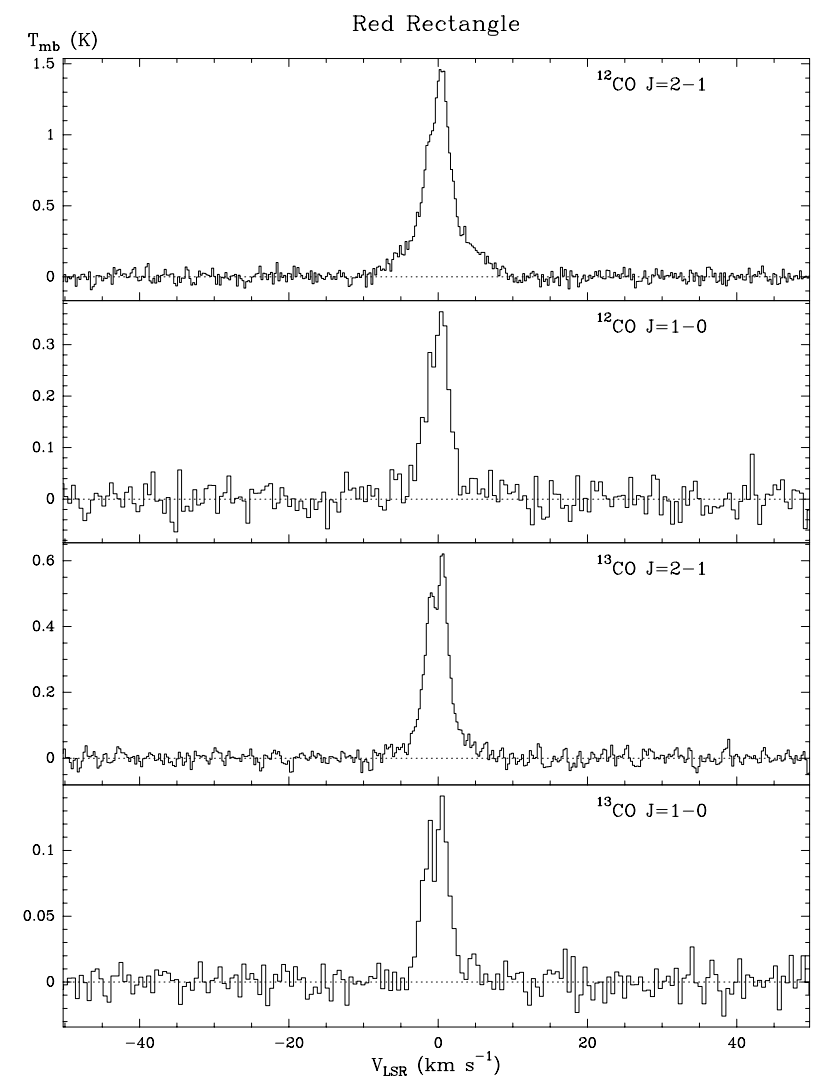

Fig. 2. Observed profiles in the Red Rectangle.

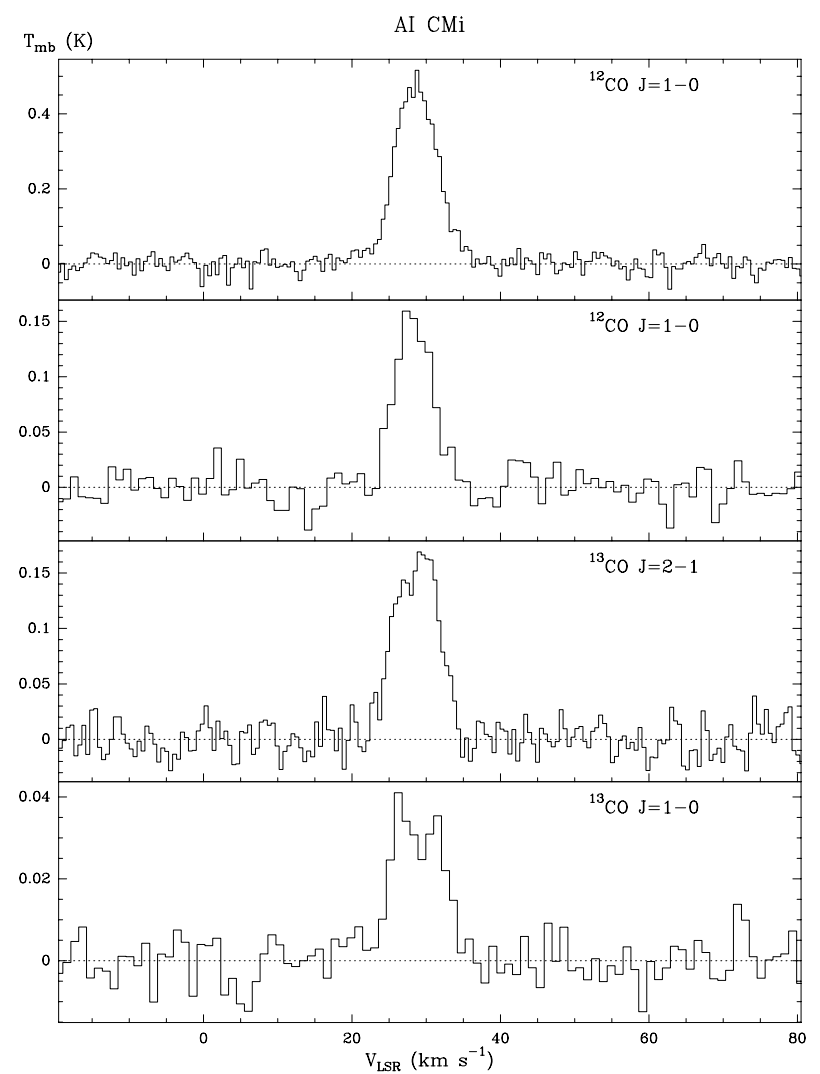

Fig. 3. Observed profiles in AI CMi.

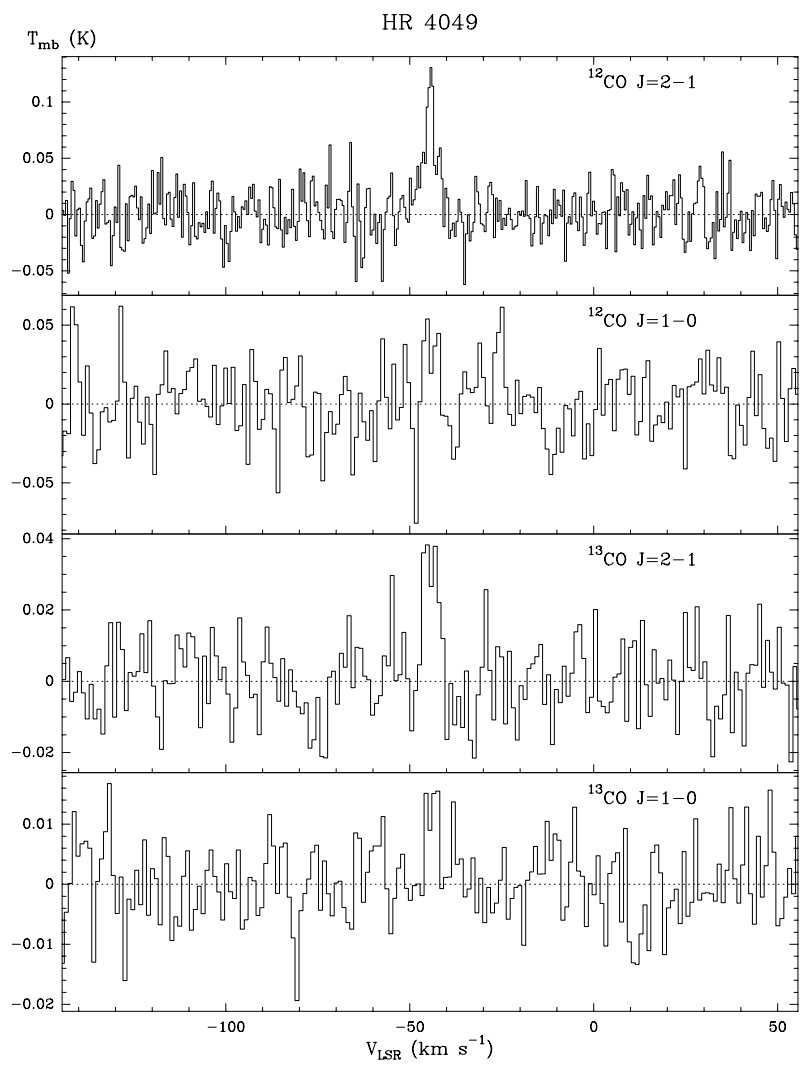

Fig. 4. Observed profiles in HR 4049.

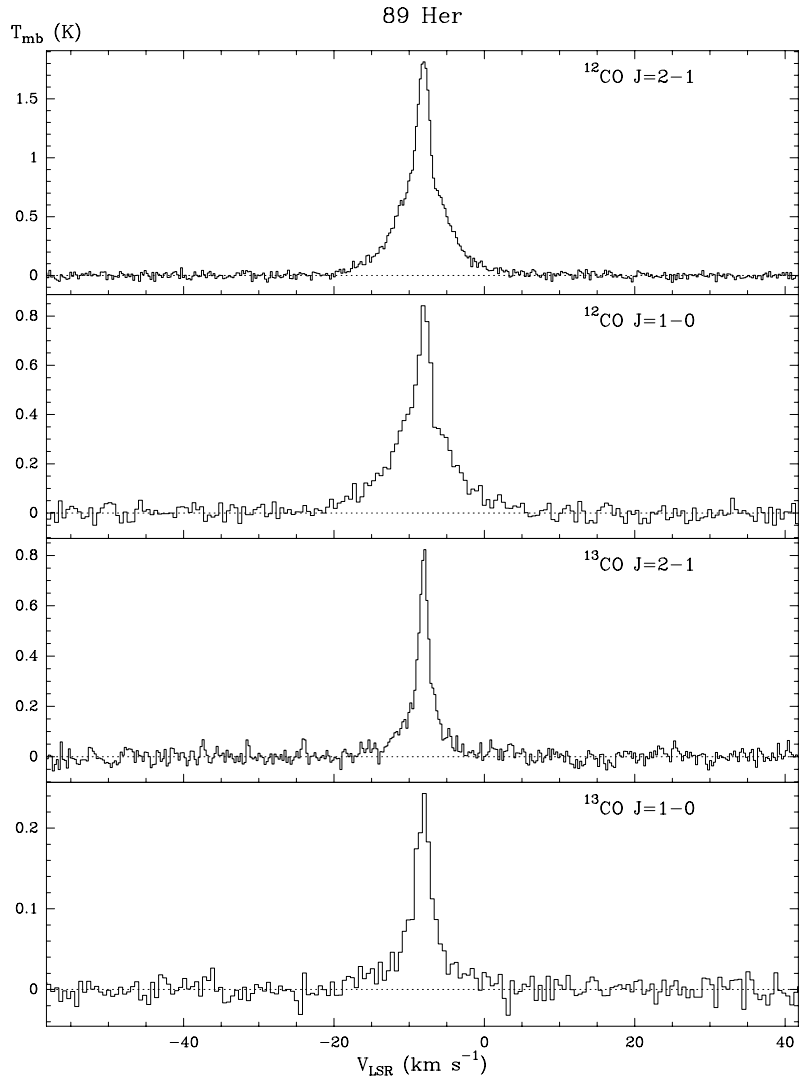

Fig. 5. Observed profiles in 89 Her. 
A\&A 557, A104 (2013)

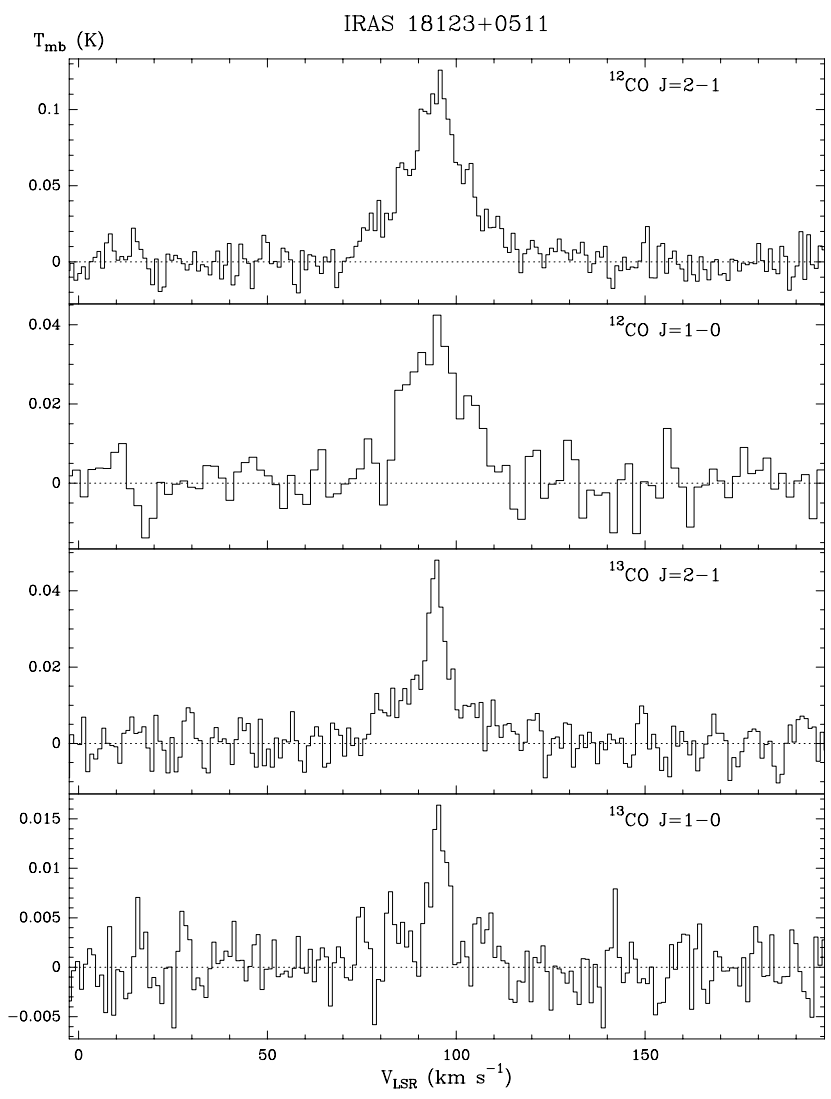

Fig. 6. Observed profiles in IRAS 18123+0511.

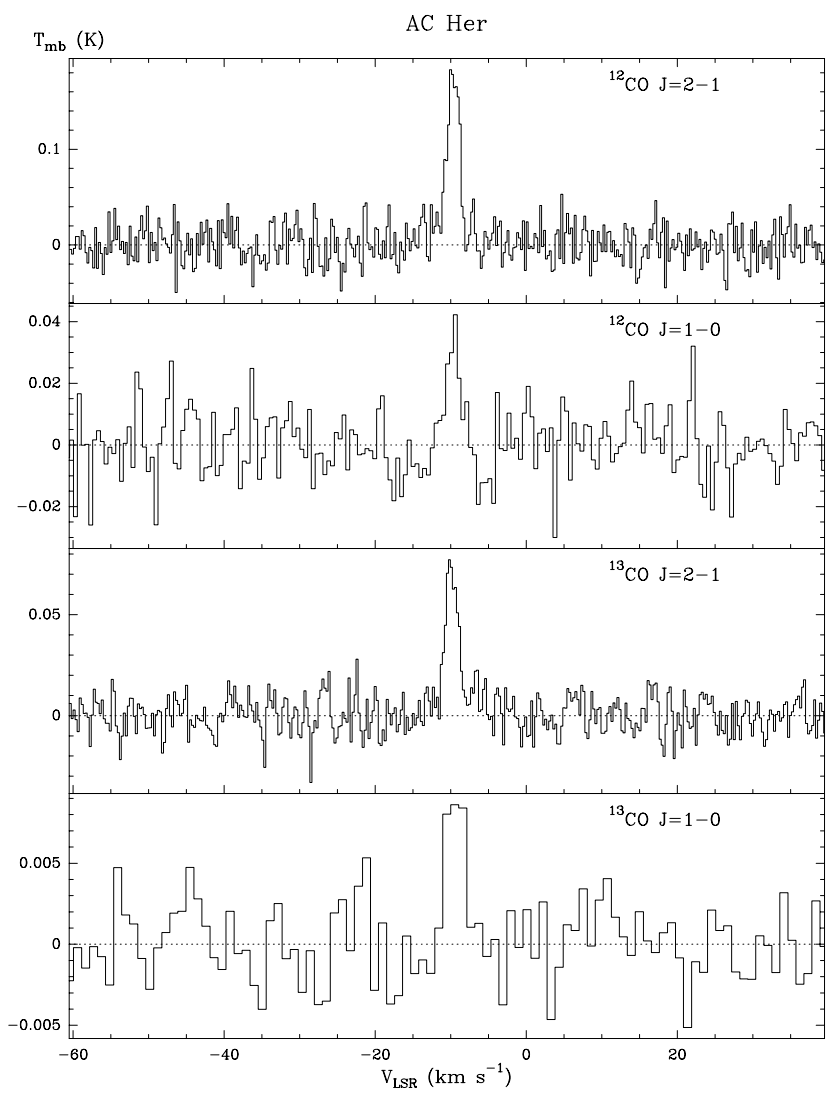

Fig. 7. Observed profiles in AC Her.

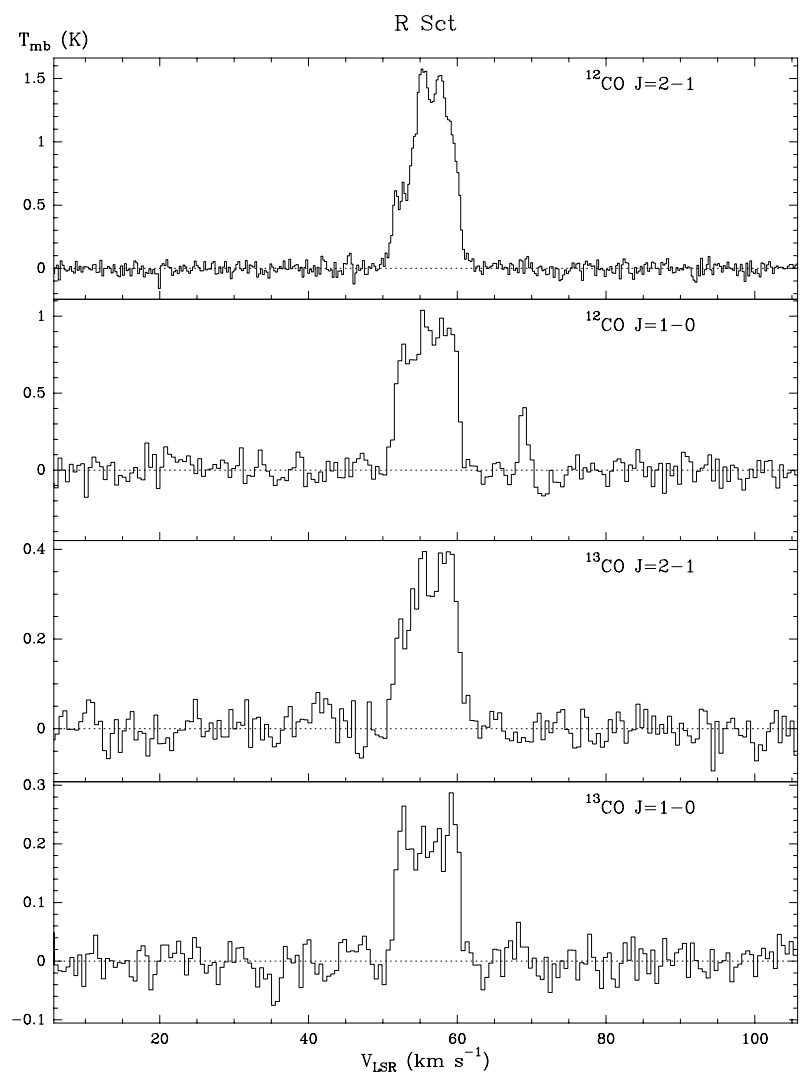

Fig. 8. Observed profiles in R Sct. The feature at about $70 \mathrm{~km} \mathrm{~s}^{-1} \mathrm{LSR}$ in ${ }^{12} \mathrm{CO} J=1-0$ is very likely to be interstellar contamination.

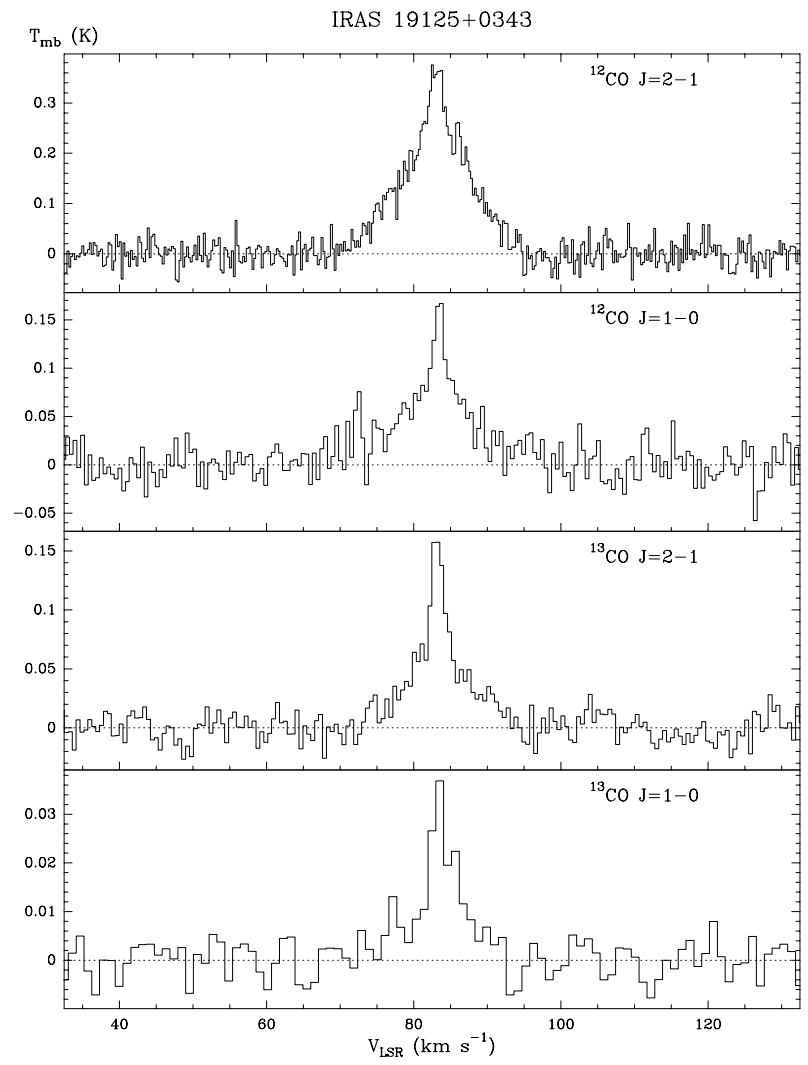

Fig. 9. Observed profiles in IRAS 19125+0343. 


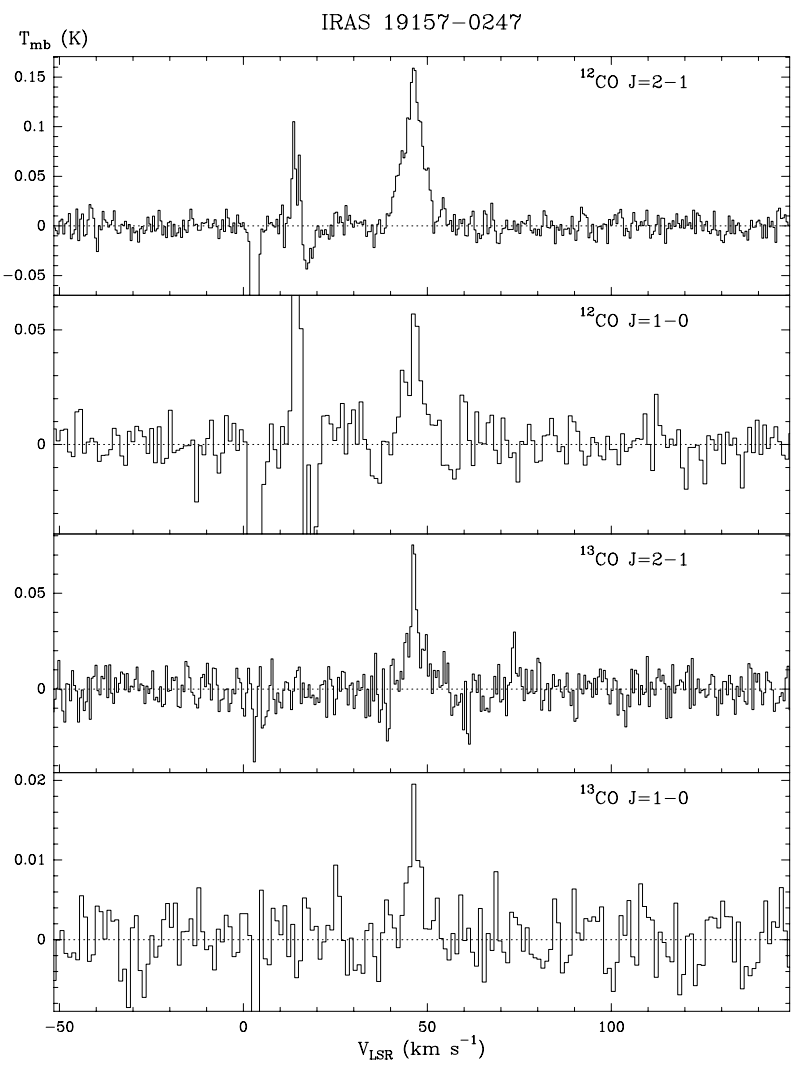

Fig. 10. Observed profiles in IRAS 19157-0247. We note the presence of interstellar contamination between 0 and $20 \mathrm{~km} \mathrm{~s}^{-1}$ LSR

For the profile peaks of the same lines in NGC 7027, we adopted the values $25 \mathrm{~K}, 1.05 \mathrm{~K}, 11.9 \mathrm{~K}$, and $0.27 \mathrm{~K}$. We also considered previous observations of CRL 618 and 89 Her (Bujarrabal et al. 2001); we note that more recent observations tend to indicate that the $1 \mathrm{~mm}$ data in that paper are probably overestimated by about a $20 \%$. After these corrections and considering variations we have found between observations in these runs and our experience with previous data, we believe that the absolute incertitude in fluxes is approximately $\pm 20 \%$.

Baselines of degree 1 to 3 were subtracted from our spectra. The resulting line profiles are shown in Figs. 1 to 11 for the sources in which emission was detected. The spectra for undetected objects are presented in Appendix A. A summary of the observational parameters is shown in Table 3: velocity range occupied by the emission (or the whole analyzed band for undetected sources), spectral resolution used to determine the tabulated parameters, peak intensity, rms noise, line area and uncertainty, and equivalent width; some comments are also given. The equivalent width is defined as the profile area divided by the peak. The noise in the line area is estimated as the rms noise multiplied by the adopted equivalent width and divided by $\sqrt{n_{\mathrm{ch}}}$, where $n_{\text {ch }}$ is the number of velocity channels within the equivalent width (i.e. the equivalent width divided by the considered spectral resolution). We note that the uncertainty in the baseline retrieval can yield noticeable (but difficult to quantify) errors in the line area in some cases.

The velocity ranges given in Table 3 for detected sources are those occupied by the full line profile and are used in particular to calculate the line area. For nondetections, the velocity range is the total range considered to estimate the upper limits and are centered on the stellar velocities (with respect to the local standard of rest, LSR) deduced from optical data (Tables 1

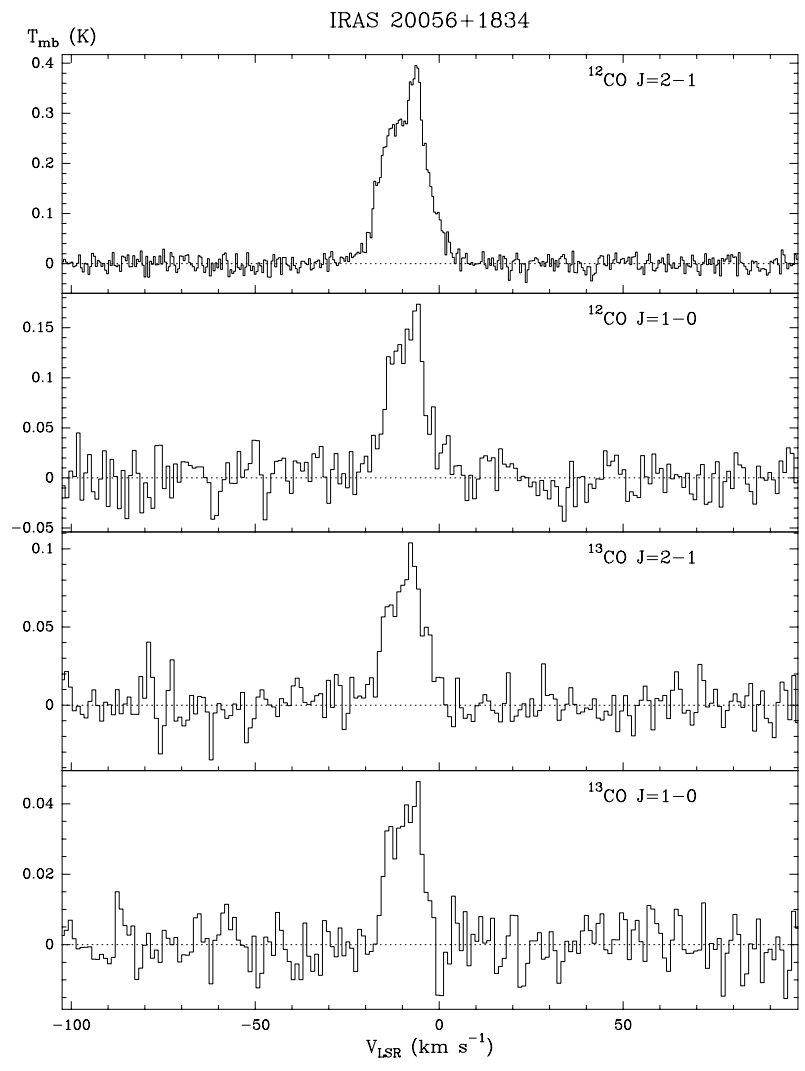

Fig. 11. Observed profiles in IRAS $20056+1834$.

and 2). The LSR peak velocity of the detected lines is found to be coincident with that of previous detections or, in the case of new detections, with the stellar velocity deduced from optical data (within $\pm 5 \mathrm{~km} \mathrm{~s}^{-1}$ ). For IRAS 20056+1834, which shows an asymmetrical profile, the stellar velocity deduced from the optical $\left(\sim 0 \mathrm{~km} \mathrm{~s}^{-1}\right)$ is uncertain but close to the velocity of the line peak.

\subsection{APEX data}

The data for the southern sources sample have been obtained using the APEX ${ }^{1} 12 \mathrm{~m}$ telescope in April-May 2012. The precipitable water vapor during the run ranged between 1.0 and $2.5 \mathrm{~mm}$ in general.

We have observed the ${ }^{12} \mathrm{CO} J=2-1$ and $J=3-2$ lines using the SHeFI APEX Band 1 (Vassilev et al. 2008) and Band 2 (Risacher et al. 2006) receivers, respectively, with both connected to the Fast Fourier Transform Spectrometer XFFTS, Klein et al. (2012). The observation was done in the standard ON-OFF mode using the wobbling secondary. In this mode, the secondary mirror of the telescope wobbles at the frequency of $1 \mathrm{~Hz}$ between the ON and OFF positions. At the same time, the telescope nods such that the OFF positions are alternatively taken at both sides of the source always at $\pm 50^{\prime \prime}$ in azimuth.

The SHeFI receivers can only be used in one band at a time, so the observation of the two lines in each object are

1 The Atacama Pathfinder EXperiment, APEX, is a collaboration between Max Planck Institut für Radioastronomie (MPIfR, 50\%), Onsala Space Observatory (OSO, 23\%), and the European Southern Observatory (ESO, 27\%) to construct and operate a modified ALMA prototype antenna as a single dish on the high-altitude site of Llano Chajnantor. 
A\&A 557, A104 (2013)

Table 3. Summary of observational results for northern sources (IRAM $30 \mathrm{~m}$ telescope).

\begin{tabular}{|c|c|c|c|c|c|c|c|c|c|}
\hline Source & Transition & $\begin{array}{l}\text { Velocity range } \\
\mathrm{km} \mathrm{s}^{-1} \text { (LSR) }\end{array}$ & $\begin{array}{l}\text { sp. resol. } \\
\mathrm{km} \mathrm{s}^{-1}\end{array}$ & $\begin{array}{c}T_{\mathrm{mb}}(\text { peak }) \\
\mathrm{K} \\
\end{array}$ & $\begin{array}{c}\mathrm{rms} \\
\mathrm{K}\end{array}$ & $\begin{array}{c}\text { Area } \\
\mathrm{K} \mathrm{km} \mathrm{s}^{-1} \\
\end{array}$ & $\begin{array}{l}\mathrm{rms}(\text { area }) \\
\mathrm{K} \mathrm{km} \mathrm{s}^{-1}\end{array}$ & $\begin{array}{l}\text { Eq. line width } \\
\mathrm{km} \mathrm{s}^{-1}\end{array}$ & Comments \\
\hline RV Tau & $\begin{array}{l}{ }^{12} \mathrm{CO} 2-1 \\
{ }^{12} \mathrm{CO} 1-0 \\
{ }^{13} \mathrm{CO} 2-1 \\
{ }^{13} \mathrm{CO} 1-0 \\
\end{array}$ & $\begin{array}{c}17 \pm 200 \\
17 \pm 200 \\
17 \pm 100 \\
17 \pm 200 \\
\end{array}$ & $\begin{array}{l}0.51 \\
0.51 \\
0.53 \\
0.53 \\
\end{array}$ & & $\begin{array}{c}2 \times 10^{-2} \\
3.5 \times 10^{-2} \\
2.5 \times 10^{-2} \\
1.5 \times 10^{-2} \\
\end{array}$ & $<6 \times 10^{-2}$ & & $5^{*}$ & $\begin{array}{l}\text { undetected } \\
\mathrm{CO} \text { emission }\end{array}$ \\
\hline DY Ori & $\begin{array}{l}{ }^{12} \mathrm{CO} 2-1 \\
{ }^{12} \mathrm{CO} 1-0 \\
{ }^{13} \mathrm{CO} 2-1 \\
{ }^{13} \mathrm{CO} 1-0 \\
\end{array}$ & $\begin{array}{l}-13 \pm 5 \\
-13 \pm 5 \\
-13 \pm 5 \\
-13 \pm 5 \\
\end{array}$ & $\begin{array}{l}1.02 \\
1.02 \\
1.06 \\
1.06 \\
\end{array}$ & $\begin{array}{l}4.5 \times 10^{-2} \\
3.1 \times 10^{-2} \\
2.7 \times 10^{-2} \\
<1 \times 10^{-2}\end{array}$ & $\begin{array}{l}1 \times 10^{-2} \\
1 \times 10^{-2} \\
1 \times 10^{-2} \\
4 \times 10^{-3} \\
\end{array}$ & $\begin{array}{c}0.28 \\
0.15 \\
0.15 \\
<3 \times 10^{-2} \\
\end{array}$ & $\begin{array}{c}3 \times 10^{-2} \\
2.5 \times 10^{-2} \\
2.5 \times 10^{-2} \\
1.0 \times 10^{-2} \\
\end{array}$ & $\begin{array}{l}6.2 \\
5.5 \\
5.6 \\
6^{*} \\
\end{array}$ & \\
\hline Red Rectangle & $\begin{array}{l}{ }^{12} \mathrm{CO} 2-1 \\
{ }^{12} \mathrm{CO} 1-0 \\
{ }^{13} \mathrm{CO} 2-1 \\
{ }^{13} \mathrm{CO} 1-0 \\
\end{array}$ & $\begin{array}{l}0 \pm 12 \\
0 \pm 12 \\
0 \pm 12 \\
0 \pm 12 \\
\end{array}$ & $\begin{array}{l}0.25 \\
0.51 \\
0.27 \\
0.53 \\
\end{array}$ & $\begin{array}{l}1.43 \\
0.34 \\
0.61 \\
0.13 \\
\end{array}$ & $\begin{array}{l}3.5 \times 10^{-2} \\
2.5 \times 10^{-2} \\
1.5 \times 10^{-2} \\
9.5 \times 10^{-3} \\
\end{array}$ & $\begin{array}{l}7.00 \\
1.53 \\
2.43 \\
0.47 \\
\end{array}$ & $\begin{array}{c}4 \times 10^{-2} \\
4 \times 10^{-2} \\
2 \times 10^{-2} \\
1.5 \times 10^{-2} \\
\end{array}$ & $\begin{array}{c}4.9 \\
4.5 \\
4.0 \\
3.65 \\
\end{array}$ & \\
\hline U Mon & $\begin{array}{l}{ }^{12} \mathrm{CO} 2-1 \\
{ }^{12} \mathrm{CO} 1-0 \\
{ }^{13} \mathrm{CO} 2-1 \\
{ }^{13} \mathrm{CO} 1-0 \\
\end{array}$ & $\begin{array}{l}9 \pm 200 \\
9 \pm 200 \\
9 \pm 100 \\
9 \pm 200 \\
\end{array}$ & $\begin{array}{l}0.51 \\
0.51 \\
0.53 \\
0.53 \\
\end{array}$ & & $\begin{array}{c}1.5 \times 10^{-2} \\
2 \times 10^{-2} \\
2 \times 10^{-2} \\
1 \times 10^{-2} \\
\end{array}$ & $<5 \times 10^{-2}$ & & $5^{*}$ & $\begin{array}{l}\text { undetected } \\
\mathrm{CO} \text { emission }\end{array}$ \\
\hline $\mathrm{AI} C \mathrm{CMi}$ & $\begin{array}{l}{ }^{12} \mathrm{CO} 2-1 \\
{ }^{12} \mathrm{CO} 1-0 \\
{ }^{13} \mathrm{CO} 2-1 \\
{ }^{13} \mathrm{CO} 1-0 \\
\end{array}$ & $\begin{array}{l}29 \pm 10 \\
29 \pm 10 \\
29 \pm 10 \\
29 \pm 10 \\
\end{array}$ & $\begin{array}{l}0.25 \\
0.51 \\
0.53 \\
1.06 \\
\end{array}$ & $\begin{array}{c}0.50 \\
0.16 \\
0.18 \\
3.5 \times 10^{-2} \\
\end{array}$ & $\begin{array}{c}3.5 \times 10^{-2} \\
2 \times 10^{-2} \\
1.5 \times 10^{-2} \\
5 \times 0^{-3}\end{array}$ & $\begin{array}{c}3.43 \\
0.95 \\
1.4 \\
0.31 \\
\end{array}$ & $\begin{array}{c}4.5 \times 10^{-2} \\
3.5 \times 10^{-2} \\
3 \times 10^{-2} \\
1.5 \times 10^{-2} \\
\end{array}$ & $\begin{array}{l}6.9 \\
5.9 \\
7.7 \\
8.8 \\
\end{array}$ & \\
\hline HR 4049 & $\begin{array}{l}{ }^{12} \mathrm{CO} 2-1 \\
{ }^{12} \mathrm{CO} 1-0 \\
{ }^{13} \mathrm{CO} 2-1 \\
{ }^{13} \mathrm{CO} 1-0 \\
\end{array}$ & $\begin{array}{l}-45 \pm 6 \\
-45 \pm 6 \\
-45 \pm 6 \\
-45 \pm 6 \\
\end{array}$ & $\begin{array}{l}0.51 \\
1.02 \\
1.06 \\
1.06 \\
\end{array}$ & $\begin{array}{c}0.11 \\
\sim 4 \times 10^{-2} \\
3.0 \times 10^{-2} \\
\sim 1.6 \times 10^{-2} \\
\end{array}$ & $\begin{array}{l}2 \times 10^{-2} \\
2 \times 10^{-2} \\
1 \times 10^{-2} \\
6 \times 10^{-3} \\
\end{array}$ & $\begin{array}{c}0.50 \\
\sim 0.1 \\
0.15 \\
5.4 \times 10^{-2} \\
\end{array}$ & $\begin{array}{c}3 \times 10^{-2} \\
4 \times 10^{-2} \\
2.5 \times 10^{-2} \\
1.5 \times 10^{-2} \\
\end{array}$ & $\begin{array}{l}4.5 \\
3.3 \\
5.7 \\
4.9 \\
\end{array}$ & \\
\hline $89 \mathrm{Her}$ & $\begin{array}{l}{ }^{12} \mathrm{CO} 2-1 \\
{ }^{12} \mathrm{CO} 1-0 \\
{ }^{13} \mathrm{CO} 2-1 \\
{ }^{13} \mathrm{CO} 1-0 \\
\end{array}$ & $\begin{array}{l}-8 \pm 14 \\
-8 \pm 14 \\
-8 \pm 14 \\
-8 \pm 14 \\
\end{array}$ & $\begin{array}{l}0.25 \\
0.51 \\
0.27 \\
0.53 \\
\end{array}$ & $\begin{array}{l}1.78 \\
0.81 \\
0.80 \\
0.23 \\
\end{array}$ & $\begin{array}{c}2.5 \times 10^{-2} \\
2.5 \times 10^{-2} \\
2.5 \times 10^{-2} \\
1 \times 10^{-2} \\
\end{array}$ & $\begin{array}{l}9.06 \\
5.01 \\
2.34 \\
0.97 \\
\end{array}$ & $\begin{array}{c}2.5 \times 10^{-2} \\
4 \times 10^{-2} \\
2 \times 10^{-2} \\
1.5 \times 10^{-2} \\
\end{array}$ & $\begin{array}{l}5.1 \\
6.2 \\
2.9 \\
4.2 \\
\end{array}$ & \\
\hline IRAS $18123+0511$ & $\begin{array}{l}{ }^{12} \mathrm{CO} 2-1 \\
{ }^{12} \mathrm{CO} 1-0 \\
{ }^{13} \mathrm{CO} 2-1 \\
{ }^{13} \mathrm{CO} 1-0\end{array}$ & $\begin{array}{l}95 \pm 25 \\
95 \pm 25 \\
95 \pm 25 \\
95 \pm 25 \\
\end{array}$ & $\begin{array}{l}0.51 \\
0.51 \\
0.53 \\
0.53 \\
\end{array}$ & $\begin{array}{c}0.12 \\
4.3 \times 10^{-2} \\
4.8 \times 10^{-2} \\
1.5 \times 10^{-2} \\
\end{array}$ & $\begin{array}{c}1.5 \times 10^{-2} \\
1 \times 10^{-2} \\
6.5 \times 10^{-3} \\
4 \times 10^{-3} \\
\end{array}$ & $\begin{array}{l}2.16 \\
0.71 \\
0.53 \\
0.14 \\
\end{array}$ & $\begin{array}{c}4 \times 10^{-2} \\
3.5 \times 10^{-2} \\
1.5 \times 10^{-2} \\
9 \times 10^{-3} \\
\end{array}$ & $\begin{array}{c}18.0 \\
16.5 \\
11.1 \\
9.3 \\
\end{array}$ & \\
\hline AC Her & $\begin{array}{l}{ }^{12} \mathrm{CO} 2-1 \\
{ }^{12} \mathrm{CO} 1-0 \\
{ }^{13} \mathrm{CO} 2-1 \\
{ }^{13} \mathrm{CO} 1-0 \\
\end{array}$ & $\begin{array}{l}-10 \pm 5 \\
-10 \pm 5 \\
-10 \pm 5 \\
-10 \pm 5 \\
\end{array}$ & $\begin{array}{l}0.25 \\
0.51 \\
0.27 \\
1.06 \\
\end{array}$ & $\begin{array}{c}0.17 \\
3.2 \times 10^{-2} \\
7.2 \times 10^{-2} \\
8.0 \times 10^{-3} \\
\end{array}$ & $\begin{array}{c}2 \times 10^{-2} \\
1 \times 10^{-2} \\
9.5 \times 10^{-3} \\
2.5 \times 10^{-3} \\
\end{array}$ & $\begin{array}{c}0.44 \\
5.5 \times 10^{-2} \\
0.21 \\
2.5 \times 10^{-2} \\
\end{array}$ & $\begin{array}{c}1.5 \times 10^{-2} \\
1 \times 10^{-2} \\
9 \times 10^{-3} \\
4.5 \times 10^{-3} \\
\end{array}$ & $\begin{array}{l}2.6 \\
1.8 \\
2.9 \\
3.0 \\
\end{array}$ & \\
\hline R Sct & $\begin{array}{l}{ }^{12} \mathrm{CO} 2-1 \\
{ }^{12} \mathrm{CO} 1-0 \\
{ }^{13} \mathrm{CO} 2-1 \\
{ }^{13} \mathrm{CO} 1-0\end{array}$ & $\begin{array}{l}56 \pm 8 \\
56 \pm 8 \\
56 \pm 8 \\
56 \pm 8 \\
\end{array}$ & $\begin{array}{l}0.25 \\
0.51 \\
0.27 \\
0.53 \\
\end{array}$ & $\begin{array}{l}1.54 \\
0.98 \\
0.39 \\
0.26 \\
\end{array}$ & $\begin{array}{c}4 \times 10^{-2} \\
6.5 \times 10^{-2} \\
5 \times 10^{-2} \\
2.5 \times 10^{-2} \\
\end{array}$ & $\begin{array}{l}10.2 \\
7.40 \\
2.84 \\
1.82 \\
\end{array}$ & $\begin{array}{l}5 \times 10^{-2} \\
1 \times 10^{-1} \\
7 \times 10^{-2} \\
5 \times 10^{-2} \\
\end{array}$ & $\begin{array}{l}6.6 \\
7.6 \\
7.4 \\
7.0 \\
\end{array}$ & $\begin{array}{l}\text { composite } \\
\text { profiles }\end{array}$ \\
\hline IRAS $19125+0343$ & $\begin{array}{l}{ }^{12} \mathrm{CO} 2-1 \\
{ }^{12} \mathrm{CO} 1-0 \\
{ }^{13} \mathrm{CO} 2-1 \\
{ }^{13} \mathrm{CO} 1-0\end{array}$ & $\begin{array}{l}83 \pm 15 \\
83 \pm 15 \\
83 \pm 15 \\
83 \pm 15 \\
\end{array}$ & $\begin{array}{l}0.25 \\
0.51 \\
0.27 \\
0.53 \\
\end{array}$ & $\begin{array}{c}0.35 \\
0.15 \\
0.15 \\
3.8 \times 10^{-2} \\
\end{array}$ & $\begin{array}{l}2.5 \times 10^{-2} \\
1.5 \times 10^{-2} \\
1.5 \times 10^{-2} \\
6.5 \times 10^{-3} \\
\end{array}$ & $\begin{array}{l}3.31 \\
1.31 \\
0.89 \\
0.21 \\
\end{array}$ & $\begin{array}{c}3.5 \times 10^{-2} \\
3.5 \times 10^{-2} \\
2 \times 10^{-2} \\
1 \times 10^{-2} \\
\end{array}$ & $\begin{array}{l}9.5 \\
8.7 \\
5.9 \\
5.5 \\
\end{array}$ & \\
\hline IRAS 19157-0247 & $\begin{array}{l}{ }^{12} \mathrm{CO} 2-1 \\
{ }^{12} \mathrm{CO} 1-0 \\
{ }^{13} \mathrm{CO} 2-1 \\
{ }^{13} \mathrm{CO} 1-0 \\
\end{array}$ & $\begin{array}{l}46 \pm 7 \\
46 \pm 7 \\
46 \pm 7 \\
46 \pm 7 \\
\end{array}$ & $\begin{array}{l}0.51 \\
1.02 \\
0.53 \\
1.06 \\
\end{array}$ & $\begin{array}{c}0.15 \\
5.1 \times 10^{-2} \\
7.0 \times 10^{-2} \\
1.6 \times 10^{-2} \\
\end{array}$ & $\begin{array}{c}9 \times 10^{-3} \\
8 \times 10^{-3} \\
8.5 \times 10^{-3} \\
3.5 \times 10^{-3} \\
\end{array}$ & $\begin{array}{c}0.99 \\
0.34 \\
0.25 \\
6.0 \times 10^{-2} \\
\end{array}$ & $\begin{array}{c}1.5 \times 10^{-2} \\
2.1 \times 10^{-2} \\
1 \times 10^{-2} \\
7 \times 10^{-3} \\
\end{array}$ & $\begin{array}{l}6.6 \\
6.5 \\
3.6 \\
3.8 \\
\end{array}$ & \\
\hline IRAS $20056+1834$ & $\begin{array}{l}{ }^{12} \mathrm{CO} 2-1 \\
{ }^{12} \mathrm{CO} 1-0 \\
{ }^{13} \mathrm{CO} 2-1 \\
{ }^{13} \mathrm{CO} 1-0 \\
\end{array}$ & $\begin{array}{l}-9 \pm 20 \\
-9 \pm 20 \\
-9 \pm 20 \\
-9 \pm 20 \\
\end{array}$ & $\begin{array}{l}0.25 \\
0.51 \\
0.53 \\
0.53 \\
\end{array}$ & $\begin{array}{c}0.39 \\
1.8 \times 10^{-2} \\
9.5 \times 10^{-2} \\
4.5 \times 10^{-2} \\
\end{array}$ & $\begin{array}{c}2 \times 10^{-2} \\
2.5 \times 10^{-2} \\
1.5 \times 10^{-2} \\
1 \times 10^{-2} \\
\end{array}$ & $\begin{array}{l}4.91 \\
1.85 \\
1.08 \\
0.42 \\
\end{array}$ & $\begin{array}{c}3.5 \times 10^{-2} \\
6 \times 10^{-2} \\
4 \times 10^{-2} \\
2 \times 10^{-2} \\
\end{array}$ & $\begin{array}{c}12.6 \\
10.2 \\
11.4 \\
9.3 \\
\end{array}$ & $\begin{array}{l}\text { composite } \\
\text { profiles }\end{array}$ \\
\hline R Sge & $\begin{array}{l}{ }^{12} \mathrm{CO} 2-1 \\
{ }^{12} \mathrm{CO} 1-0 \\
{ }^{13} \mathrm{CO} 2-1 \\
{ }^{13} \mathrm{CO} 1-0\end{array}$ & $\begin{array}{l}28 \pm 200 \\
28 \pm 200 \\
28 \pm 100 \\
28 \pm 200\end{array}$ & $\begin{array}{l}0.51 \\
0.51 \\
0.53 \\
0.53\end{array}$ & & $\begin{array}{c}1 \times 10^{-2} \\
2.5 \times 10^{-2} \\
1.5 \times 10^{-2} \\
1 \times 10^{-2}\end{array}$ & $<5 \times 10^{-2}$ & & $5^{*}$ & $\begin{array}{l}\text { undetected } \\
\mathrm{CO} \text { emission }\end{array}$ \\
\hline
\end{tabular}

Notes. The velocity range is that occupied by the full line profile and is used in particular to calculate the line area. For undetected sources, the velocity range is the total analyzed range (centered on the velocity of the source deduced from optical data). The uncertainty of the area has been calculated considering the number of spectral channels included in the equivalent line width (given in the last column, see text). An asterisk indicates values assumed from data of other lines. Uncertain values are indicated by the symbol $\sim$. 
Table 4. Summary of APEX observations of southern sources.

\begin{tabular}{|c|c|c|c|c|c|c|c|c|c|}
\hline Source & Transition & $\begin{array}{l}\text { Velocity range } \\
\mathrm{km} \mathrm{s}^{-1}(\mathrm{LSR})\end{array}$ & $\begin{array}{l}\text { sp. resol. } \\
\mathrm{km} \mathrm{s}^{-1}\end{array}$ & $\begin{array}{c}T_{\mathrm{mb}}(\text { peak }) \\
\mathrm{K}\end{array}$ & $\begin{array}{c}\mathrm{rms} \\
\mathrm{K}\end{array}$ & $\begin{array}{c}\text { Area } \\
\mathrm{K} \mathrm{km} \mathrm{s}^{-1}\end{array}$ & $\begin{array}{l}\mathrm{rms}(\text { area }) \\
\mathrm{K} \mathrm{km} \mathrm{s}^{-1}\end{array}$ & $\begin{array}{l}\text { Eq. line width } \\
\mathrm{km} \mathrm{s}^{-1}\end{array}$ & Comments \\
\hline \multirow[t]{2}{*}{ AR Pup } & ${ }^{12} \mathrm{CO} \mathrm{3-2}$ & $29 \pm 200$ & 0.53 & & 0.02 & & & & ISM narrow line \\
\hline & ${ }^{12} \mathrm{CO} 2-1$ & $29 \pm 200$ & 0.6 & & 0.06 & & & & ISM narrow line \\
\hline \multirow[t]{2}{*}{ IRAS 08544-4431 } & ${ }^{12} \mathrm{CO} 3-2$ & $45 \pm 12$ & 0.53 & 0.56 & 0.02 & 4.22 & 0.035 & 7.5 & \\
\hline & ${ }^{12} \mathrm{CO} 2-1$ & & & 0.45 & & 3 & & & SEST data (Maas et al.) \\
\hline \multirow[t]{2}{*}{ IW Car } & ${ }^{12} \mathrm{CO} \mathrm{3-2}$ & $-28 \pm 9$ & 0.53 & 0.22 & 0.03 & 0.71 & 0.035 & 3.2 & \\
\hline & ${ }^{12} \mathrm{CO} 2-1$ & $-28 \pm 9$ & 0.6 & 0.14 & 0.015 & 0.54 & 0.025 & 3.9 & \\
\hline \multirow[t]{2}{*}{ IRAS 10174-5704 } & ${ }^{12} \mathrm{CO} 3-2$ & $3 \pm 200$ & 0.53 & & 0.04 & & & & strong IS cont. \\
\hline & ${ }^{12} \mathrm{CO} 2-1$ & $3 \pm 200$ & 0.6 & & 0.02 & & & & strong IS cont. \\
\hline \multirow[t]{2}{*}{ IRAS 10456-5712 } & ${ }^{12} \mathrm{CO} 3-2$ & $-9 \pm 200$ & 0.53 & & 0.03 & & & & strong IS cont. \\
\hline & ${ }^{12} \mathrm{CO} 2-1$ & $-9 \pm 200$ & 0.6 & & 0.015 & & & & strong IS cont. \\
\hline \multirow[t]{2}{*}{ HD 95767} & ${ }^{12} \mathrm{CO} \mathrm{3-2}$ & $-32 \pm 50$ & 1.1 & 0.05 & 0.013 & 0.26 & 0.03 & 5.2 & \\
\hline & ${ }^{12} \mathrm{CO} 2-1$ & $-32 \pm 50$ & 1 & $\sim 0.03$ & 0.01 & $\sim 0.06$ & 0.02 & $5.2^{*}$ & \\
\hline \multirow[t]{2}{*}{ RU Cen } & ${ }^{12} \mathrm{CO} \mathrm{3-2}$ & $-35 \pm 200$ & 0.53 & & 0.02 & & & & undetected $\mathrm{CO}$ em. \\
\hline & ${ }^{12} \mathrm{CO} 2-1$ & $-35 \pm 200$ & 0.6 & & 0.015 & & & & undetected $\mathrm{CO}$ em. \\
\hline HD 108015 & ${ }^{12} \mathrm{CO} 2-1$ & $-2 \pm 6$ & 0.6 & 0.11 & 0.02 & 0.72 & 0.04 & 6.5 & \\
\hline \multirow[t]{2}{*}{ IRAS 15469-5311 } & ${ }^{12} \mathrm{CO} 3-2$ & $-14 \pm 200$ & 0.53 & & 0.02 & & & & strong IS cont. \\
\hline & ${ }^{12} \mathrm{CO} 2-1$ & $-14 \pm 200$ & 0.6 & & 0.02 & & & & strong IS cont. \\
\hline \multirow[t]{2}{*}{ IRAS 15556-5444 } & ${ }^{12} \mathrm{CO} 3-2$ & $0 \pm 200$ & 0.53 & & 0.02 & & & & IS contamination \\
\hline & ${ }^{12} \mathrm{CO} 2-1$ & $0 \pm 200$ & 0.6 & & 0.015 & & & & IS contamination \\
\hline
\end{tabular}

Notes. The velocity range is that occupied by the full line profile and used in particular to calculate the line area. For undetected sources, the velocity range is the total analyzed range (centered on the velocity of the source deduced from optical data). The uncertainty of the area has been calculated considering the number of spectral channels included in the equivalent line width (given in the last column, see text). An asterisk indicates value assumed from data of other lines. Uncertain values are indicated by the symbol $\sim$.

not simultaneous. For the $J=2-1$ line, the XFFTS was configured to work with 13107 channels of $0.099 \mathrm{~km} \mathrm{~s}^{-1}(76.3 \mathrm{kHz})$ of resolution, covering a band of about $1300 \mathrm{~km} \mathrm{~s}^{-1}$. For the $J=3-2$ transition, the number of channels was 6553 with a resolution of $0.13 \mathrm{~km} \mathrm{~s}^{-1}(152.6 \mathrm{kHz})$, providing a total bandwidth of $866 \mathrm{~km} \mathrm{~s}^{-1}$. The data are originally calibrated in terms of $T_{\mathrm{a}}^{*}$, using the standard procedure of observing hot and cold loads and the blank sky.

The data have been inspected and, after removing bad scans, time-averaged. Baselines of at most degree two have been removed using the ranges of the spectrum free from line features. The resolution of the APEX spectra have been downgraded to about $0.5 \mathrm{~km} \mathrm{~s}^{-1}$ or $1.0 \mathrm{~km} \mathrm{~s}^{-1}$ by averaging adjacent channels. Finally, the data have been rescaled into $T_{\mathrm{mb}}$ units, using the values for the telescope efficiencies given by Güsten et al.(2006). See a summary of the results in Table 4 . We give more details on the data analysis in Appendix B, where the resulting APEX spectra are shown (Figs. B.1 to B.10).

In more than one half of the sources, we note that the spectra show contamination due to emission of interstellar gas in the direction of the source and of the reference (OFF) position. In some cases, this contamination does not harm our observation, but it is so strong and widespread around the systemic velocity in other sources that we can not conclude on the presence of any circumstellar contribution. These cases are noted in Table 4 . The presence of this contamination is not a surprise, since many of the selected sources are located very close to the galactic plane.

\section{Rotating disks in the observed sources}

We have observed 24 post-AGB stars in which the presence of inner, very compact rotating disks was suspected from the presence of a significant NIR excess (other properties of the sources are mentioned in Sects. 1 and 2). Fourteen sources were observed with the $30 \mathrm{~m}$ IRAM telescope in ${ }^{12} \mathrm{CO}$ and
${ }^{13} \mathrm{CO} J=2-1$ and $J=1-0$ transitions, and ten southern objects were observed with APEX in ${ }^{12} \mathrm{CO} J=3-2$ and $J=2-1$. The detection rate is very high: of the 14 sources observed with the $30 \mathrm{~m}$ telescope, we detected $\mathrm{CO}$ emission in 11 . We also detected disk emission from 4 southern post-AGB stars. Because of the lower signal-to-noise ratio $(\mathrm{S} / \mathrm{N})$ ratio attained with the APEX telescope and the presence of significant contamination by interstellar emission in many sources (Sect. 3.2, Appendix B), any estimate of a detection rate comparable to that deduced for the northern sources is impossible.

Only four of these sources were previously detected in CO lines: the Red Rectangle, 89 Her, R Sct, and IRAS 085444431 , with a tentative detection of AC Her. The line parameters obtained in those observations are similar to those found here but with significantly less complete and accurate profiles in all cases. See previous molecular line data in Bujarrabal et al. (2005, 2007), Jura et al. (1995), Alcolea \& Bujarrabal (1995), Maas et al. (2003), and references therein.

The Red Rectangle, the best studied source in our sample, shows the characteristic profiles of rotating disks, which are narrow and with a central peak or two nearby peaks, as seen in Fig. 2. This kind of line shape is often observed in disks around young stars and has been very well studied. It is widely accepted from both observational and theoretical grounds that these profiles are a good probe of the presence of disks in rotation (Guilloteau \& Dutrey 1998; Guilloteau et al. 2013; Bujarrabal \& Alcolea 2013, etc.). The CO line profiles observed in evolved nebulae of other classes are very different. In AGB stars, the profiles are wide (with a width often over $20 \mathrm{~km} \mathrm{~s}^{-1}$ ) with two horns or parabolic. In other standard PPNe, the lines are even broader (with profiles sometimes as wide as $100 \mathrm{~km} \mathrm{~s}^{-1}$ ) and show a core+wings structure. Interferometric maps of the Red Rectangle (Bujarrabal et al. 2005) conclusively confirmed that a rotating disk is the dominant nebular component in mm-wave line emission. The maps show that the CO-rich disk is extended 
(about $6^{\prime \prime}$ in diameter, equivalent to about $6 \times 10^{16} \mathrm{~cm}$, and $\lesssim 1^{\prime \prime}$ in width), obviously in rotation, and placed in the equator of the optical nebula. High-J Herschel/HIFI observations show an excess in the line wings that seems impossible to explain from disk emission alone, this excess is also present though less clearly in low- $J$ transitions; see data and detailed models in Bujarrabal \& Alcolea (2013). According to these authors, this excess probably comes from a relatively diffuse bipolar component expanding at about $10 \mathrm{~km} \mathrm{~s}^{-1}$. This feature is conspicuous in the ${ }^{12} \mathrm{CO}$ lines but very weak in ${ }^{13} \mathrm{CO}$ lines, which indicates that the $\mathrm{CO}$ emission from the bipolar outflow is likely to be optically thin and that the rare-isotope lines would only come from the disk. These suggestions have been confirmed by very recent ALMA maps of ${ }^{12} \mathrm{CO}$ and ${ }^{13} \mathrm{CO}$ lines (Bujarrabal et al. 2013). Therefore, emission from a bipolar outflow significantly contributes to the wings of the profiles shown here, mostly to those of ${ }^{12} \mathrm{CO}$.

89 Her has also been mapped in CO lines (Bujarrabal et al. 2007). An expanding double-bubble is responsible for most emission at velocities separated by more than $3-4 \mathrm{~km} \mathrm{~s}^{-1}$ from the line center. There is also a practically unresolved component in the center that is very likely to be a rotating disk, in view of the low velocities involved and the very similar single-dish $\mathrm{CO}$ profiles shown by 89 Her and the Red Rectangle (Figs. 2 and 5).

In both the Red Rectangle and 89 Her, the mass of the outflow is lower than that contained in the disk, which is the dominant nebular component. In any case, the total mass of the rotating or expanding gas around these stars remains moderate, of $\lesssim 10^{-2} M_{\odot}$. See Sect. 5 for more details.

From the rest of the northern sources detected here, six (DY Ori, HR 4049, IRAS 18123+0511, AC Her, IRAS 19125+0343, and IRAS 19157-0247) show narrow profiles that are very similar to those of the Red Rectangle and 89 Her, and two (R Sct and IRAS 20056+1834) show composite profiles including a narrow component similar to the main one found in the other sources. In AI CMi, the profiles are narrow but less sharp than in the Red Rectangle or 89 Her. AI CMi shows OH emission with a twohorn profile similar to those typical of AGB stars, except for the very low expansion velocity (and the high polarization degree in $1612 \mathrm{MHz}$ emission, which is typical of post-AGB $\mathrm{OH}$ emitters, Wolak et al. 2012). OH emission expands between 24 and $33 \mathrm{~km} \mathrm{~s}^{-1}$ LSR, similar to our CO lines. The narrow profiles and low-intensity wings of our CO lines in AI CMi strongly suggest emission from a rotating disk, which may be superimposed on emission from an expanding component. The line wings of IRAS $18123+0511$ and IRAS $19125+0343$ are conspicuous around a prominent central component. These wings are particularly intense in the ${ }^{12} \mathrm{CO} J=2-1$ line, the most opaque of our lines, but much less evident in ${ }^{13} \mathrm{CO}$ emission. In these two sources, we find therefore a dominant disk emission plus, probably, a significant contribution from an expanding component. The profiles of R Sct are complex; the central spike that may be due to disk emission just represents a fraction of the total intensity. IRAS $20056+1834$ also shows complex profiles with a narrow peak. The uncertain stellar velocity of IRAS $20056+1834$ deduced from studies in the optical, $\sim 0 \mathrm{~km} \mathrm{~s}^{-1}$, is close to the velocity of the narrow peak but more positive than the centroid of the line-wings, which appear clearly blue-shifted. We note that the outflow feature does not become relatively weaker for low-opacity lines in these two sources and unlike the case of the objects discussed above.

We therefore conclude that the molecule-rich nebula is dominated by an extended rotating disk in 9 sources (the Red Rectangle, 89 Her, DY Ori, HR 4049, IRAS 18123+0511, AC Her, IRAS 19125+0343, IRAS 19157-0247, and probably
AI CMi), out of a total of 14 sources in our sample of sources that were accurately observed with the 30-telescope. The Red Rectangle and 89 Her show intense line wings that are known from high-resolution maps to come from low-mass bipolar outflows with velocities of $5-10 \mathrm{~km} \mathrm{~s}^{-1}$. Two of the other objects in this group, IRAS $18123+0511$ and IRAS $19125+0343$, also show intense line wings at velocities of $\pm 5-10 \mathrm{~km} \mathrm{~s}^{-1}$ from the center that very probably come from a low-mass bipolar outflow similar to those of the Red Rectangle and 89 Her. AI CMi could also show emission from expanding gas, in view of the relatively wide lines found in this source. These slow bipolar outflows would then be associated with the presence of equatorial disks in rotation. We recall that bipolar outflows are found in many standard PPNe (see Sect. 1), although they show much higher masses and velocities than those found here (Sect. 5).

Two other objects, R Sct and IRAS 20056+1834, show complex lines with a narrow component (about $5 \mathrm{~km} \mathrm{~s}^{-1}$ wide), which are different than those discussed in the previous paragraph. We suggest that this narrow feature comes from a rotating disk that only contains a fraction of the total mass of these nebulae. The rest of the $\mathrm{CO}$ emission detected in these two objects (whose line are in total $10-15 \mathrm{~km} \mathrm{~s}^{-1}$ wide) would probably come from low-velocity outflows.

Of the ten southern sources observed with APEX, four clearly show disk-like CO profiles: IRAS 08544-4431, IW Car, HD 95767, and HD 108015. The intense ${ }^{12} \mathrm{CO} J=3-2$ line of IRAS 08544-4431 (Fig. B.2) presents wide wings that suggest a contribution of an expanding component. The ${ }^{12} \mathrm{CO} J=2-1$ and $J=1-0$ lines observed by Maas et al. (2003) in this source show similar characteristics. As mentioned, the lower quality of the observations of these southern sources prevents an analysis of the data as deep as for the northern sources.

\section{Estimates of the rotating disk mass in post-AGB objects}

\subsection{Basic relations between the total emitting gas mass and the observed line intensity}

From our observational results, it is possible to systematically derive estimates of the total mass of molecule-rich gas in the studied nebula. If we assume that the emission is optically thin, that the emitting region is much smaller than the telescope resolution and that some characteristic excitation state can represent the whole emission of the disk, the total mass is proportional to the velocity-integrated main-beam temperature.

Optically thin ${ }^{13} \mathrm{CO}$ lines are expected, in view of the significantly low ${ }^{13} \mathrm{CO} /{ }^{12} \mathrm{CO}$ line ratio, for mostly the $J=1-0$ transition (but the ${ }^{13} \mathrm{CO} /{ }^{12} \mathrm{CO}$ intensity ratio is usually found to be higher than the expected abundance ratios, suggesting optically thick ${ }^{12} \mathrm{CO}$ emission, particularly in the line core). Our detailed calculations for the case of the Red Rectangle (Bujarrabal \& Alcolea 2013) also indicate optically thin ${ }^{13} \mathrm{CO}$ lines. Note that ${ }^{13} \mathrm{CO}$ lines are also preferable to estimate the mass of the rotating disks because the contribution of the eventual expanding components (as found in the well studied cases, Sects. 1 and 4) is much smaller and probably negligible in many cases. We note that the $J=1-0$ transition is a priori expected to be less opaque than the $J=2-1$ one, because a) both transitions are easily excited (the upper level of ${ }^{13} \mathrm{CO} J=2-1$ is just at $16 \mathrm{~K}$ from the ground); b) the low Einstein A-coefficients, $A(1-0)=6.3 \times 10^{-8} \mathrm{~s}^{-1}$, guarantee an easy thermalization of 
the low- $J$ lines (see calculations in e.g. Bujarrabal et al. 2005; Bujarrabal \& Alcolea 2013); and c) the opacity of the rotational line is roughly proportional to $J^{2}$ under the above conditions.

The assumption of an emitting region that is smaller than the telescope beam is likely to be satisfied in our case, particularly for the $J=1-0$ lines, since the size of the CO images obtained in 89 Her and the Red Rectangle (which are the strongest emitters among our sources and the only ones for which good maps have been obtained, Sects. 1 and 4 ), are $\sim 6^{\prime \prime}$, and the beam half-power total widths are about $12^{\prime \prime}$ at $1 \mathrm{~mm}$ wavelength and about $23^{\prime \prime}$ at $3 \mathrm{~mm}$. We have also seen in Sect. 4 that the ${ }^{13} \mathrm{CO}$ emission of our sources is more compact, since the emission of the disk is not contaminated by components in expansion.

Under the above conditions, the observed main-beam temperature of a given transition is given by

$T_{\mathrm{mb}}\left(V_{\mathrm{LSR}}\right)=\frac{h v}{k}\left[\frac{1}{\mathrm{e}^{h v / k T_{\mathrm{ex}}}-1}-\frac{1}{\mathrm{e}^{h v / k T_{\mathrm{BG}}}-1}\right] \tau \phi\left(V_{\mathrm{LSR}}\right) \frac{\Omega_{\mathrm{s}}}{\Omega_{\mathrm{mb}}}$

where $\Omega_{\mathrm{mb}}$ and $\Omega_{\mathrm{s}}$ are, respectively, the solid angles subtended by the telescope beam and the source (we assume $\Omega_{\mathrm{s}}<\Omega_{\mathrm{mb}}$ ), $T_{\mathrm{ex}}$ and $T_{\mathrm{BG}}$ are the excitation temperature of the transition and the background temperature (we assume $T_{\mathrm{ex}} \sim T_{\mathrm{k}}$, the kinetic temperature, and $\left.T_{\mathrm{BG}}=2.7 \mathrm{~K}\right), v$ is the frequency of the transition, and $\phi\left(V_{\mathrm{LSR}}\right)$ is the normalized line profile in terms of Doppler-shifted velocity (LSR frame). The other symbols have their usual meaning.

The opacity is

$\tau=\frac{c^{3}}{8 \pi v^{3}} A_{u, l} g_{u}\left(x_{l}-x_{u}\right) n_{\mathrm{tot}} X(\mathrm{CO}) L$.

Where the subindexes $l$ and $u$ represent, respectively, the lower and upper level of the transition, $g$ is the statistical weight of a level, $x$ is the relative population per magnetic sublevel (where $n$ is the relative level population, or $x=n / g), n_{\text {tot }}$ is the total number density of particles, $X(\mathrm{CO})$ is the abundance of ${ }^{12} \mathrm{CO}$ or ${ }^{13} \mathrm{CO}$ relative to the total number of particles, and $L$ is the typical length of the source along the line of sight (perpendicular to the plane of the sky). This expression is perhaps not the most usual one of the optical depth; it is used because the profile is given in terms of velocity and not of frequency. We recall that the definition of $T_{\mathrm{ex}}$ is $x_{u} / x_{l}=\mathrm{e}^{-h v / k T_{\mathrm{ex}}}$.

Converting the source solid angle (in arcsec units) into source surface $\left(\mathrm{cm}^{2}\right)$, the velocity integrated main-beam temperature becomes

$$
\int T_{\mathrm{mb}} \mathrm{d} V \propto\left[\frac{1}{\mathrm{e}^{h v / k T_{\mathrm{ex}}}-1}-\frac{1}{\mathrm{e}^{h v / k T_{\mathrm{BG}}}-1}\right]\left(x_{l}-x_{u}\right) X M_{\mathrm{mol}} D^{2},
$$

where $M_{\text {mol }}$ is the total mass of the molecule-rich emitting gas (i.e. the characteristic total density multiplied by the source volume) and $D$ is the distance to the object. We can thus estimate the total mass from the observed profile area for optically thin, unextended emission. The proportionality factor is straightforward from Eqs. (1) and (2).

We note that in Eq. (3)

$$
\frac{1}{\mathrm{e}^{h v / k T_{\mathrm{ex}}}-1}=\frac{x_{u}}{x_{l}-x_{u}}
$$

and

$$
x_{l, u} \sim \mathrm{e}^{-E_{l, u} / T_{\text {rot }}} / F\left(T_{\text {rot }}\right),
$$

where $E$ is the energy in temperature units of the considered levels (in our case, $J=0,1$, or 2) and $F$ is the partition function for a representative rotational temperature, $T_{\text {rot }}$, that describes the population of the whole rotational ladder. For an actual value of the partition function, $T_{\text {rot }}$ is defined as the temperature that gives that value if we assume thermalization of the level populations. For a simple rotational ladder and in the limit of $T_{\text {rot }}$ much higher than the separation of the low levels, $F\left(T_{\text {rot }}\right)=T_{\text {rot }} / B_{\text {rot }}$, where $B_{\text {rot }}$ is the rotational constant of the molecule (for ${ }^{13} \mathrm{CO}$, $\left.B_{\text {rot }}=2.6 \mathrm{~K}\right)$. In our calculations, we always used the exact expression:

$$
F\left(T_{\text {rot }}\right)=\sum_{J} g_{j} \mathrm{e}^{-E_{j} / T_{\text {rot }}} .
$$

We cannot assume that $T_{\text {rot }} \sim T_{\mathrm{k}}\left(\sim T_{\text {ex }}\right.$ for low- $J$ transitions $)$ in our case (with relatively high values of $T_{\mathrm{k}}$ ). The low- $J$ levels joined by the transitions that we are dealing with are easily populated, but the population of high- $J$ levels is probably strongly subthermal, so for them $x_{j} / x_{0}<\mathrm{e}^{-E_{j} / T_{\mathrm{k}}}$ and $T_{\text {rot }}<T_{\mathrm{k}}$. We will see later estimates of the values that we can expect for $T_{\text {rot }}$. Considering that $T_{\mathrm{BG}} \ll T_{\text {ex }}$ and $E_{u} \ll T_{\text {ex }}$ for our lines, the dependence of the total mass on $T_{\text {ex }}$ practically vanishes and the dependence of the total mass estimate on the level excitation is basically given by the rotational temperature.

\subsection{Estimates of the CO rotational temperature in post-AGB rotating disks}

The temperature of the molecular gas in the Red Rectangle was first estimated by Bujarrabal et al. (2005) from maps of CO mmwave lines, in which obtained values were between about 70 and $30 \mathrm{~K}$. However, those low- $J$ transitions are not good tools to estimate the excitation of such a warm gas. Later, Herschel/HIFI observations of the $J=6-5, J=10-9$, and $J=16-15$ transitions were used to deduce that the gas temperature in the disk of the Red Rectangle must be about two times higher (Bujarrabal et al. 2012; Bujarrabal \& Alcolea 2013).

The temperature of the molecular (compact) disk of $89 \mathrm{Her}$ was deduced to be $\gtrsim 60 \mathrm{~K}$ from mm-wave maps (Bujarrabal et al. 2007). Herschel observations of 89 Her, R Sct, and IRAS $19125+0511$ yield high- $J$ transitions that are weaker by at least a factor 3 than expected from the low- $J$ line ratios found here and the Herschel/HIFI data of the Red Rectangle (Teyssier et al., in prep.; Bujarrabal \& Alcolea 2013). This result strongly suggests that the temperatures are clearly lower in these sources than in the Red Rectangle. Comparison of the Herschel data of 89 Her, R Sct, and IRAS 19125+0511 with theoretical line ratios by Bujarrabal et al. (2012), which are calculated under general conditions, indicates temperatures lower than $100 \mathrm{~K}$. Therefore, we can expect $60 \mathrm{~K} \lesssim T_{\mathrm{k}} \lesssim 100 \mathrm{~K}$ in $89 \mathrm{Her}$, and similar relations probably also hold for R Sct and IRAS 19125+0511.

For the Red Rectangle, typical densities of about $10^{4}-10^{5} \mathrm{~cm}^{-3}$ were deduced (Bujarrabal \& Alcolea 2013). The densities are probably higher in 89 Her, which is more intense than the Red Rectangle even though it is placed at a larger distance, but most of the other sources observed here are significantly weaker.

For these temperature and density values, we can expect that the low- $J$ transitions are thermalized but not for $J \gtrsim 5$, which show significantly higher Einstein coefficients ( $A$ is roughly proportional to $J^{3}$ ). The populations of these high- $J$ levels, which significantly contribute to the partition function for the relatively high temperatures that we are dealing with, cannot be neglected. Therefore, statistical equilibrium calculations of the level populations are necessary to estimate the values of the equivalent $T_{\text {rot }}$ that must be used to calculate the partition function. 


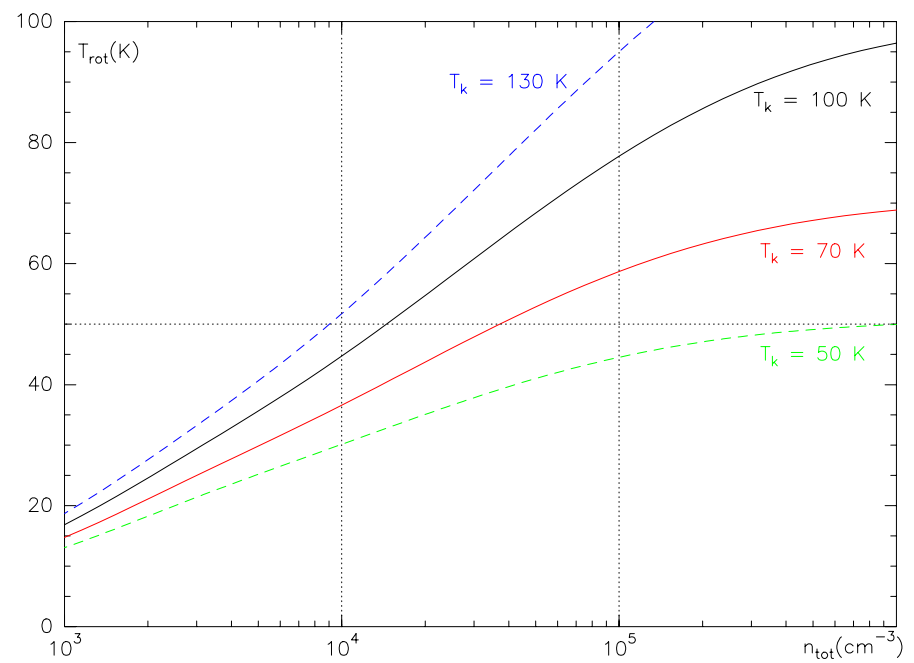

Fig. 12. Estimates of the equivalent rotational temperature describing the partition function for a variety of densities and temperatures. The most probable ranges are of about $10^{4}-10^{5} \mathrm{~cm}^{-3}$ for the densities (dashed vertical lines) and of about 70-100 K for the temperatures (continuous lines).

We have performed calculations of $T_{\text {rot }}$ for a variety of densities and temperatures in the optically thin limit. We recall that these values represent the temperature that the gas should have in the case of level thermalization yielding the same partition function as that actually given by the calculations. In the optically thin limit, the details of the radiative transfer treatment are not relevant to the estimate of the level population (which are given by a simple noniterative calculation). We used collisional rates from the $\lambda$-database (Schöier et al. 2005; Yang et al. 2010). The presence of continuum sources has no significant effect in the level population for the case that we are describing (Bujarrabal \& Alcolea 2013; Santander-García et al. 2013). In our simple case, $T_{\text {rot }}$ depends only on the density and the temperature.

Our calculations are shown in Fig. 12. For the expected ranges of kinetic temperatures (between approximately $70 \mathrm{~K}$ and $100 \mathrm{~K}$ ) and of total densities (between approximately $10^{4}-10^{5} \mathrm{~cm}^{-3}$ ), we expect a rotational temperature $T_{\text {rot }} \sim 50 \mathrm{~K}$. The uncertainty of this value is less than a factor two, which results in a similar uncertainty for the partition function. We will accordingly adopt $T_{\text {rot }}=50 \mathrm{~K}$ in our calculations of the disk mass from the observed line intensities.

When ${ }^{13} \mathrm{CO} J=1-0$ cannot be used (e.g. because of very weak emission), our formulae can be applied to data of other lines. In that case, we must note that we can underestimate the mass because, as mentioned (Sect. 5.1), the ${ }^{12} \mathrm{CO}$ lines and the ${ }^{13} \mathrm{CO} J=2-1$ line are more optically thick. Moreover, the $J=2-1$ lines are probably not less extended, because of the moderate excitation required and their higher opacities, so the smaller $J=2-1$ beam (12-13") can miss some emission. Remember that the effect of the source extent on the measured intensity is noticeable when the half-power beam width is not much larger than the source size. In all objects in which the exercise can be done, the estimates from ${ }^{13} \mathrm{CO} J=1-0$ are larger than those from the other lines, proving that these later computations really underestimate the actual mass value.

\subsection{The mass of the disks rotating around post-AGB stars}

We use Eq. (3) (Sect. 5.1) to estimate the mass of the rotating disks that we have found in our sample of post-AGB stars. We assume: $T_{\text {rot }}=50 \mathrm{~K}$ (Sect. 5.2), $X\left({ }^{13} \mathrm{CO}\right)=2 \times 10^{-5}$ (see
Table 5. Values of the nebular mass derived from our data.

\begin{tabular}{lcccl}
\hline \hline Source & $\begin{array}{c}\text { Mass } \\
M_{\odot}\end{array}$ & \multicolumn{2}{c}{ Typical size } & Comments \\
& \multicolumn{1}{c}{$\mathrm{cm}$} & \\
\hline RV Tau & $<8 \times 10^{-3}$ & $<0.5$ & $<1.3 \times 10^{16}$ & \\
DY Ori & $2 \times 10^{-3}$ & 0.37 & $1.1 \times 10^{16}$ & \\
Red Rectangle & $6.6 \times 10^{-3}$ & & & total neb. \\
Red Rectangle & $6 \times 10^{-3}$ & 2 & $2.3 \times 10^{16}$ & disk \\
U Mon & $<9 \times 10^{-4}$ & $<0.4$ & $<5 \times 10^{15}$ & \\
AI CMi & $1.9 \times 10^{-2}$ & 1.2 & $2.7 \times 10^{16}$ & prob. exp. comp. \\
HR 4049 & $6.3 \times 10^{-4}$ & 0.6 & $6 \times 10^{15}$ & \\
89 Her & $2.7 \times 10^{-2}$ & & & total neb. \\
89 Her & $1.4 \times 10^{-2}$ & 1.5 & $2.3 \times 10^{16}$ & disk \\
IRAS 18123+0511 & $4.7 \times 10^{-2}$ & 0.6 & $3 \times 10^{16}$ & prob. exp. comp. \\
AC Her & $8.4 \times 10^{-4}$ & 0.7 & $1.1 \times 10^{16}$ & \\
R Sct & $5 \times 10^{-2}$ & & & total neb. (complex prof.) \\
R Sct & $\sim 6.9 \times 10^{-3}$ & $\sim 1$ & $\sim 1.5 \times 10^{16}$ & disk \\
IRAS 19125+0343 & $1.3 \times 10^{-2}$ & 1 & $2.3 \times 10^{16}$ & prob. exp. comp. \\
IRAS 19157-0247 & $1.4 \times 10^{-2}$ & 0.7 & $3 \times 10^{16}$ & \\
IRAS 20056+1834 & $10^{-1}$ & & & total neb. (complex prof.) \\
IRAS 20056+1834 & $\sim 2.5 \times 10^{-2}$ & $\sim 0.6$ & $\sim 1.7 \times 10^{16}$ & disk \\
R Sge & $<9 \times 10^{-3}$ & $<0.3$ & $<7 \times 10^{15}$ & \\
\hline IRAS 08544-4431 & $\sim 7.7 \times 10^{-3}$ & 2.2 & $1.8 \times 10^{16}$ & from ${ }^{12} \mathrm{CO}$ data \\
IW Car & $\sim 5.3 \times 10^{-3}$ & 1.3 & $2 \times 10^{16}$ & from ${ }^{12} \mathrm{CO}$ data \\
HD 95767 & $\sim 1.2 \times 10^{-3}$ & 0.6 & $1.3 \times 10^{16}$ & from ${ }^{12} \mathrm{CO}$ data \\
HD 108015 & $\sim 2.3 \times 10^{-2}$ & 1.2 & $3 \times 10^{16}$ & from ${ }^{12} \mathrm{CO}$ data \\
\hline
\end{tabular}

Notes. Distance values are discussed in Sect. 2. For $89 \mathrm{Her}$, R Sct, and IRAS 20056+1834, we have calculated the total nebular mass and also the mass derived from the central line peak, expected to better represent the disk emission. See discussion on these cases and other uncertain values in Sect. 5.3 and Appendix B.

below), an average particle mass of $3 \times 10^{-24} \mathrm{~g}$, and $T_{\text {ex }}=T_{\text {rot }}$ (which has little impact on the final values, Sect. 5.2). We use the ${ }^{13} \mathrm{CO} J=1-0$ data as far as possible, which are the best for our purposes (Sects. 5.1 and 5.2). The distances adopted for each object are given in Tables 1 and 2, and the telescope beam widths are given in Sect. 3.

The results of our mass estimate are shown in Table 5. We also give comments on the estimate meaning and reliability. Some sources deserve a detailed discussion.

The rotating disk of the Red Rectangle has been well studied, as seen in Sects. 1 and 4 and in Bujarrabal et al. (2005) and Bujarrabal \& Alcolea (2013). This object has served as a prototype to allow our study of other less well-known nebulae. The mass derived in those studies, $M_{\text {mol }} \sim 6 \times 10^{-3} M_{\odot}$, is completely compatible with that obtained here $\left(6.6 \times 10^{-3} M_{\odot}\right)$. It is important to note that the contribution to the ${ }^{13} \mathrm{CO}$ low- $J$ profiles due to expanding gas components (which is obvious in ${ }^{12} \mathrm{CO}$ lines, see Sect. 4) is probably minor. Bujarrabal \& Alcolea (2013) derived a mass for the outflowing gas in this object of about $10^{-3} M_{\odot}$ from their high- $J{ }^{12} \mathrm{CO}$ profiles, which could be included in the slightly higher value of the total mass derived here.

In 89 Her, the $J=2-1$ maps show that the emission from velocities separated by more than $3-4 \mathrm{~km} \mathrm{~s}^{-1}$ from the line center comes only from expanding gas. Accordingly, with our standard estimate of the mass, we have performed another estimate retrieving a degree 2 baseline from the profiles that eliminates this spectral component. The result is also given in Table 5. Again our estimate is compatible with those deduced by Bujarrabal et al. (2007), $\sim 10^{-2} M_{\odot}$.

The mass values derived here for the Red Rectangle and 89 Her are very similar to those deduced from completely independent (and more detailed) estimates, which is a proof of the reliability of our simple method. 
IRAS $18123+0511$ and IRAS $19125+0343$ show profiles with a narrow core and line wings. The wings are relatively intense in ${ }^{12} \mathrm{CO} J=2-1$, but the profiles are much narrower in the optically thin ${ }^{13} \mathrm{CO}$ lines. It seems therefore that there is a significant contribution of an expanding component in these sources, but it is probably negligible in ${ }^{13} \mathrm{CO} J=1-0$. Thus, our standard mass calculation, only considering the contribution of the disk, is likely reliable for them.

R Sct and IRAS 20056+1834 show composite profiles that suggest that the contribution from a heavy outflow is dominant in the resulting relatively wide profiles $\left(10-15 \mathrm{~km} \mathrm{~s}^{-1}\right)$. In both cases, there is a narrow component, about $5 \mathrm{~km} \mathrm{~s}^{-1}$ wide, that could come from a rotating disk. We have performed mass calculations also for this disk component, trying to isolate the contribution of the narrow features in the observed profiles (in a similar way as for 89 Her), but the estimate of the corresponding intensity is not straightforward; values are given in Table 5. In any case, we think that those values, $7 \times 10^{-3} M_{\odot}$ for R Sct and $2.5 \times 10^{-2} M_{\odot}$ for IRAS $20056+1834$, are the best estimates of the disk mass in these nebulae. The total mass values deduced for these objects, which are close to $0.1 M_{\odot}$, are the highest in our sample, and seem dominated by the supposed expanding component. We must keep in mind that these mass values may be overestimated by about a factor 2 if the characteristic rotational temperature in the dominant expanding component is lower than that deduced here for rotating disks, as it happens for the (fast) outflowing components of more massive PPNe, in which temperatures of about $20 \mathrm{~K}$ are often found (Bujarrabal et al. 2001).

The rest of the detected sources show narrow profiles that strongly suggest that the emission from an equatorial disk dominates and that we are reasonably calculating the mass of this rotating component. In DY Ori, we do not detect the ${ }^{13} \mathrm{CO} J=1-0$ transition, deriving $M_{\mathrm{mol}}<3 \times 10^{-3} M_{\odot}$. On the other hand, we find $M_{\text {mol }} \gtrsim 1.6 \times 10^{-3} M_{\odot}$ from ${ }^{13} \mathrm{CO} J=2-1$ (see Sect. 5.2), so we can safely assign a value of $M_{\text {mol }} \sim 2 \times 10^{-3} M_{\odot}$ to this source. For RV Tau and R Sge we derive just upper limits to the molecular-gas mass; we find respectively $M_{\text {mol }}<8 \times 10^{-3} M_{\odot}$ and $M_{\text {mol }}<9 \times 10^{-3} M_{\odot}$ for these sources, which are not much smaller than the results obtained in detected sources. From the ${ }^{13} \mathrm{CO} J=1-0$ limit found in $\mathrm{U}$ Mon, which is a relatively nearby source, we deduce $M_{\text {mol }}<9 \times 10^{-4} M_{\odot}$. This is one of the lowest mass values that we have found in our sample; the lowest actual measurement is that derived for HR 4049, at a value of $6 \times 10^{-4} M_{\odot}$.

These nebular mass values are significantly lower than those found in standard PPNe, which are often $\sim 0.2-1 M_{\odot}$ (Sect. 1). This result holds even if we consider the uncertainty in the disk mass determination, which is of about a factor 2 in general cases and about a factor 3 in sources with poorly known distances (Sect. 5.3.1).

We have also given mass estimates for the outflowing components, but they are in general uncertain, as these outflows are not well studied yet. In the Red Rectangle and 89 Her, relatively good estimates of the outflowing gas mass, however, can be obtained from our calculations and previous works (Bujarrabal et al. 2007; Bujarrabal \& Alcolea 2013). For the Red Rectangle we conclude that the outflowing gas mass is about $10^{-3} M_{\odot}$, and the expansion velocity is about $10 \mathrm{~km} \mathrm{~s}^{-1}$. For $89 \mathrm{Her}$, we find a mass of about $10^{-2} M_{\odot}$ and a typical velocity of $5 \mathrm{~km} \mathrm{~s}^{-1}$. The linear momenta of the outflows in these sources is then of about $2 \times 10^{36} \mathrm{~g} \mathrm{~cm} \mathrm{~s}^{-1}$ and $10^{37} \mathrm{~g} \mathrm{~cm} \mathrm{~s}^{-1}$, respectively, and their kinetic energy is $\sim 2-5 \times 10^{42} \mathrm{erg}$. The linear momentum that can be delivered per unit time by the stellar luminosity (in all directions and neglecting photon trapping effects, i.e., $L / c$ ) is $2.5 \times 10^{34} \mathrm{~g} \mathrm{~cm} \mathrm{~s}^{-1} \mathrm{yr}^{-1}$ for the Red Rectangle and $4 \times 10^{34} \mathrm{~g} \mathrm{~cm} \mathrm{~s}^{-1} \mathrm{yr}^{-1}$ for 89 Her. (We recall that, as usual in this kind of comparisons, we are not exactly dealing with the linear momentum but with a kind of scalar linear momentum integrated in all directions). Contrary to the case of most standard PPNe (Bujarrabal et al. 2001), the linear momentum and energy carried by the bipolar outflows in these objects is very moderate and stellar radiation could provide them in a reasonable time (in comparison with the expected ejection times). We note that the mass/radiation momentum ratio is distance independent. We can reach a similar conclusion for the other objects that probably show bipolar outflows (identified from the relatively intense line wings present in at least IRAS 18123+0511, IRAS 19125+0343, AI CMi, and IRAS 08544-4431), since the outflowing gas mass and velocity in them, although less well known, cannot be significantly higher than for the Red Rectangle and 89 Her.

The momenta of the outflows of $\mathrm{R}$ Sct and IRAS 20056+1834, which seem to be the dominant nebular components, are around 5-10 times higher than for the Red Rectangle and 89 Her but are still far lower than those typical of standard PPNe (Bujarrabal et al. 2001). R Sct and IRAS $20056+1834$ are in some way intermediate between the low-luminosity post-AGB objects studied here and the (luminous and massive) standard $\mathrm{PPNe}$ and young $\mathrm{PNe}$; indeed, the luminosity of R Sct is as high as $10^{4} L_{\odot}$ (that of IRAS $20056+1834$ is not well known, Table 2). There are also PPNe with low luminosities $\left(\sim 10^{3} L_{\odot}\right)$ that do not show NIR excess nor disk-like $\mathrm{CO}$ profiles (but significantly broader ones), such as in IRAS 19500-1709 and IRAS 23304+6147, as seen in Bujarrabal et al. (2001). The nebular masses in these objects were found to be low, at a value of $\sim 10^{-2} M_{\odot}$, which is not significantly higher than those of our objects (but note that these nebulae are expanding at high velocity).

Finally, we have estimated the disk mass in the southern sources that we have observed with APEX and also using a SEST observation from Maas et al. (2003), see details in Appendix B. No ${ }^{13} \mathrm{CO}$ data are available for those sources. The mass values are then estimated after converting those derived from ${ }^{12} \mathrm{CO} J=2-1$ intensities to equivalent ${ }^{13} \mathrm{CO} J=1-0$ values, by using an empirical relation between results from both lines for northern sources observed with the $30 \mathrm{~m}$ telescope, and considering the different telescope beam widths (Sect. 3). In these calculations, we adopted a ${ }^{12} \mathrm{CO}$ abundance of $2 \times 10^{-4}$. The low isotopic abundance ratio assumed here is compatible with previous studies of theses objects (Bujarrabal et al. 1990; Bujarrabal \& Alcolea 2013); in any case, the assumed value has no effect on the final derived mass, since we used an empirical relation to obtain values equivalent to those given by ${ }^{13} \mathrm{CO}$ lines. Because of the high optical depth of ${ }^{12} \mathrm{CO}$ lines, the derivation of reliable values of the isotopic abundance ratio requires detailed modeling case by case, which is out of the scope of this paper. These approximations obviously add uncertainty to our estimates. The results are also given in Table 5 in the last four lines. We did not try to deduce upper limits for the undetected southern objects because of this uncertain conversion and the other observational problems discussed in Sect. 3.2 and Appendix B.

\subsubsection{Uncertainties in the mass estimates}

The major sources of uncertainty in our estimates come from the assumptions of $T_{\text {rot }}$, which is less than a factor of two (Sect. 5.2), and of $X\left({ }^{13} \mathrm{CO}\right)$. We think that the uncertainty in our assumption on $X\left({ }^{13} \mathrm{CO}\right)$ is also lower than a factor of two, since existing 
estimates yield values of this parameter similar to our adopted one. In AGB stars, $X\left({ }^{12} \mathrm{CO}\right)$ is found to vary between about $2-4 \times 10^{-4}$ in O-rich stars and 6-8 $\times 10^{-4}$ in carbon-rich stars (see e.g. Teyssier et al. 2006; Ramstedt et al. 2008, and references therein), but the ${ }^{12} \mathrm{CO} /{ }^{13} \mathrm{CO}$ abundance ratio is higher in carbon-rich stars, resulting in a similar value of the ${ }^{13} \mathrm{CO}$ abundance, which is usually found to be $X\left({ }^{13} \mathrm{CO}\right) \sim 2 \times 10^{-5}$ (e.g. Kahane et al. 1992, 2000; Bujarrabal et al. 1994; Schöier \& Olofsson 2000; Schöier et al. 2011, etc.). Studies of molecular gas in $\mathrm{PNe}$ and $\mathrm{PPNe}$ often give values of $X\left({ }^{12} \mathrm{CO}\right)$ that are slightly lower than for AGB stars, but the isotopic ratio is found to be also lower and values of $X\left({ }^{13} \mathrm{CO}\right) \sim 2 \times 10^{-5}$ are usually compatible with the observations (e.g. Bujarrabal et al. 2001; Bujarrabal \& Alcolea 2013; Woods et al. 2005; Soria-Ruiz et al. 2013 , etc. $)$. In particular, $X\left({ }^{13} \mathrm{CO}\right) \sim 2 \times 10^{-5}$ is the value deduced for the Red Rectangle by Bujarrabal \& Alcolea (2013) and used in their systematic study of PPNe by Bujarrabal et al. (2001). In the case that we assume a different value for $X\left({ }^{13} \mathrm{CO}\right)$, the nebular mass that we deduce here is easily scaled, following $M_{\text {mol }} \propto 1 / X\left({ }^{13} \mathrm{CO}\right)$. Both uncertainties, due to the excitation conditions and the assumed abundance, are not expected to be correlated, so we can assume a total uncertainty of about a factor 2. We must keep in mind that our estimates of the nebular mass concern only the molecule-rich component. The central stars of our sources (Tables 1 and 2) are often not hot enough to significantly photodissociate $\mathrm{CO}$, but the presence of a hotter companion or the interstellar UV field could yield photodissociation of molecules (respectively in inner and outer disk regions). Therefore, our estimates are always a lower limit to the total nebular mass, which may include components of (mostly) atomic gas with significant amounts of mass, in particular extended haloes that are not probed by our observations.

Another, sometimes difficult to quantify, source of uncertainty is the distance estimate. In the worst cases, we have deduced a distance value just assuming a typical luminosity of $3000 L_{\odot}$ (Sect. 2.1). Since the luminosity of post-AGB stars is not expected to differ by more than a factor of 2-3 from that assumed value, we expect errors in the distance by factors $\sim 1.5$ in those cases and an error in the mass not larger than a factor 2-3. Considering the sources of uncertainty mentioned above, we derive a total uncertainty in the mass determination of a factor 3-4 for objects in which the distance is poorly known.

\subsection{Rough estimates of the typical sizes of the disks}

Following a similar reasoning to those developed in previous subsections, it is possible to estimate the typical sizes of the disks responsible for the detected lines. We assume that the low- $J$ lines are thermalized and that the ${ }^{12} \mathrm{CO} J=2-1$ line is opaque. The first assumption very likely holds in view of the low Einstein coefficients of these low transitions, which are $\lesssim 10^{-6} \mathrm{~s}^{-1}$. The ${ }^{12} \mathrm{CO} J=2-1$ line is very probably opaque, since the measured ratios between this line and the more optically thin ${ }^{12} \mathrm{CO} J=1-0$ and ${ }^{13} \mathrm{CO}$ lines are much lower than expected for optically thin emissions. Under these assumptions, the main-beam brightness temperatures at the line center must be approximately equal to the ratio between the solid angles occupied by the source and the telescope beam, multiplied by the typical temperature (assumed here to be $50 \mathrm{~K}$ ). This estimate is rough because the temperature and opacity are likely to vary across the source, and, therefore, we are deriving an $a v$ erage or characteristic size, disregarding the disk inclination for instance. However, we think that it will give an idea of a parameter that is unknown for all sources, except for the Red Rectangle and 89 Her. Once we assume a value of the distance (Sect. 2, Table 1), we can also derive a typical linear size. Values derived in this way are summarized in Table 5. In the sources in which two components are considered, we only derive the size of the disk with that of the expanding component being still more uncertain. In any case, we must keep in mind that the expanding component could always contribute to the size derived in this way. Typical derived sizes range between about $0 .{ }^{\prime} 5$ for weak sources with $T_{\mathrm{mb}} \sim 0.1 \mathrm{~K}$ and $2^{\prime \prime}$ for intense sources with $T_{\mathrm{mb}} \sim 1.5 \mathrm{~K}$.

\section{Conclusions}

We have studied a sample of 24 post-AGB stars that show NIR excess indicative of the presence of hot dust, which has been proposed to be placed in an inner rotating disk. These stars are systematically binaries and often show lower luminosities and temperatures than the standard well-studied protoplanetary and young planetary nebulae.

Fourteen sources were observed with the IRAM $30 \mathrm{~m}$ telescope in the ${ }^{12} \mathrm{CO}$ and ${ }^{13} \mathrm{CO} J=2-1$ and $J=1-0$ transitions. Eleven of these objects (the Red Rectangle, 89 Her, DY Ori, HR 4049, IRAS 18123+0511, AC Her, IRAS 19125+0343, IRAS 19157-0247, AI CMi, R Sct, and IRAS 20056+1834) were clearly detected. See data in Table 3 and Figs. 1 to 11 and A.1 to A.3.

Ten southern sources were observed in the ${ }^{12} \mathrm{CO} J=3-2$ and $J=2-1$ transitions with APEX, as seen in Sect. 2.2, Appendix B, Table 4, and Figs. B.1 to B.10. Four sources (IRAS 08544-4431, IW Car, HD 95767, and HD 108015) are detected. Because of the poorer $\mathrm{S} / \mathrm{N}$ attained in this southern sample and the presence of strong interstellar contamination in several sources, the detection rate is less meaningful in this case.

Most of the observed CO lines show characteristic profiles composed of a central narrow peak and low-velocity wings, which are very similar to those expected from rotating disks and those very often observed in disks orbiting young stars, and are very different from the wide profiles found in AGB stars and other PPNe (Sect. 4). Indeed, we have found this kind of profiles in the Red Rectangle, the only post-AGB source in which the presence of a rotating equatorial disk is well proven (from highresolution mapping of $\mathrm{CO}$ emission, Bujarrabal et al. 2005). In view of the disk-like line profiles, we conclude that the dominant component in most of our nebulae is an equatorial disk in rotation (Sect. 4).

It is known that the Red Rectangle and 89 Her also show a gas component in axial expansion, occupying a bipolar structure, that is responsible (at least partially) for the relatively intense emission at LSR velocities farther than about $\pm 5 \mathrm{~km} \mathrm{~s}^{-1}$ from the systemic one (Sects. 1 and 4). AI CMi, IRAS 18123+0511, and IRAS 19125+0343 show disk-like profiles with relatively intense wings that strongly suggest that gas in expansion is also present in them. The presence of an expanding component is also probable in the southern source IRAS 08544-4431. In R Sct and IRAS $20056+1834$, the profiles are more complex but show a narrow feature, which suggests that a rotating disk is present but the total emission is dominated by that of expanding components.

We stress the high detection rate of rotating disks among the well-studied objects with the $30 \mathrm{~m}$ telescope. For the undetected sources (RV Tau, U Mon, and R Sge), we derive upper limits of the mass and size of the disks (see below) that are not much lower than the values found in the detected nebulae, strongly 
suggesting that nondetection in these cases is just due to the attained sensitivity.

We have calculated the mass of the CO-emitting gas, assuming the line emission properties expected in rotating disks, which are mostly deduced from previous studies of the Red Rectangle and 89 Her, as discussed in Sect. 5. We argue that the expected uncertainty in the mass determination probably do not exceed a factor 2 (a factor of 3-4 when the distance is very poorly known). The derivation of the mass is performed from data of ${ }^{13} \mathrm{CO} J=1-0$, when it was detected, because this line is less affected by opacity effects and because the relatively large telescope beam at this frequency prevents nondetection of extended components. For our southern sources observed with APEX, in which data of this line are not available, we derived mass estimates by using an empirical relation that we found between results obtained from ${ }^{13} \mathrm{CO} J=1-0$ and ${ }^{12} \mathrm{CO} J=2-1$ (Appendix B); we must keep in mind that these results are significantly more uncertain than for the other objects. A summary of the mass values is shown in Table 5. We tried to separate the contributions of the expanding and rotating components in some objects: the Red Rectangle and $89 \mathrm{Her}$, for which we have information on the distribution of both components from other works, and R Sct and IRAS 20056+1834, in which the probable expanding component seems dominant.

The mass of the rotating disks derived here typically ranges between $10^{-3}$ and $10^{-2} M_{\odot}$ (Sect. 5.3). Higher values are only found in objects in which a significant contribution of an expanding component is probable; for R Sct and IRAS 20056+1834, we deduce a total mass of $5 \times 10^{-2}-10^{-1} M_{\odot}$, but we note that our procedure may overestimate the mass values by up to a factor of two in these cases (since we assume emission properties typical of disks, as discussed in Sect. 5). Only HR 4049, AC Her, and $\mathrm{U}$ Mon show disk mass values moderately lower than $10^{-3} M_{\odot}$. $\mathrm{U}$ Mon was not detected, and we derive in fact an upper limit of $9 \times 10^{-4} M_{\odot}$. The mass upper limits derived for RV Tau and R Sge, our two other significant nondetections, are higher than $10^{-3} M_{\odot}$.

The total and disk mass values derived in our objects are significantly lower than those derived for standard, well-studied PPNe and PNe (CRL 2688, CRL 618, NGC 7027, etc.), which show nebular masses of about $0.2-1 M_{\odot}$ (Sect. 1, Bujarrabal et al. 2001). This result holds in spite of the uncertainties in the mass estimates. The velocities found in our objects, even those of the expanding components and considering projection effects, are always very moderate, with values of $\lesssim 10 \mathrm{~km} \mathrm{~s}^{-1}$, which are significantly lower than those usual in the above mentioned standard nebulae (often higher than $100 \mathrm{~km} \mathrm{~s}^{-1}$, Sect. 1).

Although the existing information on the expanding components is still poor, we derive low mass values for the best studied cases (the Red Rectangle and 89 Her, Sect. 5.3) between $10^{-3}$ and a $10^{-2} M_{\odot}$. The typical expansion velocities are found to range between 5 and $10 \mathrm{~km} \mathrm{~s}^{-1}$. Other objects studied here, notably AI CMi, IRAS 18123+0511, IRAS 19125+0343, and IRAS 08544-4431, probably present similar values of these parameters. Consequently, the linear momenta of the outflows in these objects are low, comparable to what stellar radiation pressure could deliver in reasonable times of a few hundred years and much lower than those of the very massive and fast outflows of standard PPNe (Bujarrabal et al. 2001). Even for R Sct and IRAS $20056+1834$, in which the outflowing component seems dominant, we derive moderate linear momentum values (Sect. 5.3). We stress that this result does not show that radiation pressure is actually powering these bipolar outflows. We recall that the studied objects present the main properties (binarity, rotating disk, and possible presence of reaccretion) that are required to explain bipolar nebulae and flows by interaction between previously ejected shells and post-AGB jets (Sect. 1). It is remarkable that there is no clear evidence to our knowledge of disks in rotation in PPNe with high luminosity and mass, although, precisely in these objects, the acceleration of their powerful outflows seems to require reaccretion from a disk.

It is possible to derive estimates of the disk extent from the ${ }^{12} \mathrm{CO} J=2-1$ main-beam brightness temperatures measured in our objects by assuming that this line is optically thick and thermalized, which are acceptable assumptions (Sect. 5, Table 5). Typical derived sizes range between about 0.5 and $2^{\prime \prime}$, equivalent to typical linear sizes between $5 \times 10^{15}$ and $3 \times 10^{16} \mathrm{~cm}$. These CO-rich disks are therefore significantly larger than the very compact disks $\left(<10^{15} \mathrm{~cm}\right)$ that are supposed to be responsible for the NIR excess of these objects, but they are much smaller than standard PPNe and young PNe, which show total sizes significantly larger than $10^{17} \mathrm{~cm}$ (e.g. Bujarrabal et al. 1988, 2001).

In summary, the high detection rate in our observations of extended rotating disks indicates that these structures are common in post-AGB stars, being present in all or almost all post-AGB sources showing a significant NIR excess (which are a good fraction of cataloged post-AGB stars, Sect. 1). Our results confirm that the compact dust components responsible for this NIR excess are in rotation and are likely to coincide with the innermost regions of the $\mathrm{CO}$ emitting disks. We derive disk (and total) mass values ranging between $10^{-3}$ and $10^{-2} M_{\odot}$, which are significantly lower than the mass of well-known standard PPNe. Two of our objects, the Red Rectangle and 89 Her, are known to also present low-velocity bipolar outflows; in a few others, our single-dish profiles strongly suggest the presence of similar components. In general, the outflows are a minor component of the total nebula, except probably in objects with complex profiles.

The main properties of the different kinds of post-AGB objects, notably the binarity and the nebular mass and dynamics, and the possible formation of planetary nebulae from them, will be the subject of a forthcoming work.

Acknowledgements. This work has been supported by the Spanish MICINN, program CONSOLIDER INGENIO 2010, grant "ASTROMOL" (CSD200900038). We are grateful to the anonymous referee of the paper for his/her helpful comments. This work has made extensive use of the SIMBAD database (http://simbad.u-strasbg.fr/simbad/sim-fid).

\section{References}

Acker, A., Boffin, H. M. J., Outters, N., et al. 2012, Rev. Mex. Astron. Astrofis., 48,223

Acke, B., Degroote, P., Lombaert, R., et al. 2013, A\&A, 551, A76

Alcock, C., Allsman, R. A., Alves, D. R., et al. 1998, AJ, 115, 1921

Alcolea, J., Neri, R., \& Bujarrabal, V. 2007, A\&A, 468, L41

Balick, B., \& Frank, A. 2002, ARA\&A, 40, 439

Bujarrabal, V., \& Alcolea, J. 2013, A\&A, 552, A116

Bujarrabal, V., Bachiller, R., Alcolea, J., \& Martin-Pintado, J. 1988, A\&A, 206, L17

Bujarrabal, V., Alcolea, J., \& Bachiller, R. 1990, A\&A, 234, 355

Bujarrabal, V., Castro-Carrizo, A., Alcolea, J., \& Sánchez Contreras, C. 2001, A\&A, 377, 868

Bujarrabal, V., Castro-Carrizo, A., Alcolea, J., \& Neri, R. 2005, A\&A, 441, 1031 Bujarrabal, V., Van Winckel, H., Neri, R., et al. 2007, A\&A, 468, L45 Bujarrabal, V., Alcolea, J., Van Winckel, H., et al. 2013, A\&A, in press Castro-Carrizo, A., Bujarrabal, V., Fong, D., et al. 2001, A\&A, 367, 674 de Ruyter, S., van Winckel, H., Dominik, C., Watqers, L. B. F. M., \& Dejonghe, H. 2005, A\&A, 435, 161

Deroo, P., van Winckel, H., Min, M., et al. 2006, A\&A, 450, 181

Fong, D., Meixner, M., Castro-Carrizo, A., et al. 2001, A\&A, 367, 652

Frank, A., \& Blackman, E. G. 2004, ApJ, 614, 737

Gielen, C., van Winckel, H., Reyniers, M., et al. 2009, A\&A, 508, 1391 
Gielen, C., Bouwman, J., van Winckel, H., et al. 2011, A\&A, 533, A99 Huggins, P. J., Bachiller, R., Cox, P., \& Forveille, T. 1996, A\&A, 315, 284 Guilloteau, S., \& Dutrey, A. 1998, A\&A, 339, 467

Guilloteau, S., Di Folco, E., Dutrey, A., et al. 2013, A\&A, 549, A92

Güsten, R., Nyman, L. A., Schilke, P., et al. 2006, A\&A, 454, L13

Kahane, C., Cernicharo, J., Gómez-González, J., \& Guélin, M. 1992, A\&A, 256, 235

Kahane, C. Dufour, E. Busso, M., et al. 2000, A\&A, 357, 669

Kimura, R. K., Gruenwald, R., \& Aleman, I. 2012, A\&A, 541, A112

Klein, B., Hochgürtel, S., Krämer, I., et al. 2012, A\&A, 542, L3

Maas, T., Van Winckel, H., Lloyd Evans, T., et al. 2003, A\&A, 405, 271

Men'shchikov, A. B., Schertl, D., Tuthill, P. G., et al. 2002, A\&A, 393, 867

Pottasch, S. R. 1984, Astrophys. Space Sci. Lib., 107

Ramstedt, S., Schöier, F., Olofsson, H., \& Lundgren, A. A. 2008, A\&A, 486, 645

Kameswara Rao, N., Goswami, A., \& Lambert, D. L. 2002, MNRAS, 334, 129

Risacher, C., Vassilev, V., Monje, R., et al. 2006, A\&A, 454, L17

Santander-García, M., Bujarrabal, V., Koning, N, \& Steffen, W. 2013, A\&A, submitted
Schöier, F. L., \& Olofsson, H. 2000, A\&A, 359, 586

Schöier, F. L., van der Tak, F. F. S., van Dishoeck, E. F., \& Black, J. H. 2005, A\&A, 432, 369

Schöier, F. L., Maercker, M., Justtanont, K., et al. 2011, A\&A, 530, A83

Soker, N. 2002, ApJ, 568, 726

Soria-Ruiz, R., Bujarrabal, V., \& Alcolea, J. 2013, A\&A, submitted

Trams, N. R., Waters, L. B. F. M., Lamers, H. J. G. L. M., et al. 1991, A\&AS, 87,361

van Aarle, E., van Winckel, H., Lloyd Evans, T., et al. 2011, A\&A, 530, A90

Van Winckel, H. 2003, ARA\&A, 41, 391

Van Winckel, H., Waelkens, C., Fernie, J. D., \& Waters, L. B. F. M. 1999, A\&A, 343, 202

Vassilev, V., Meledin, D., Lapkin, I., et al. 2008, A\&A, 490, 1157

Waters, L. B. F. M., Trams, N. R., \& Waelkens, C. 1992, A\&A, 262, L37

Wolak, P., Szymczak, M., \& Gérard, E. 2012, A\&A, 537, A5

Woods, P. M., Nyman, L.-A., \& Schöier, F. L., et al. 2005, A\&A, 429, 977

Yang, B., Stancil, P. C., Balakrishnan, N., \& Forrey, R. C. 2010, ApJ, 718, 1062

Pages 17 to 20 are available in the electronic edition of the journal at http://www . aanda. org 


\section{Appendix A: Additional $\mathbf{3 0}$ m-telescope CO data}

In this Appendix, we show our $30 \mathrm{~m}$ telescope data of RV Tau, $\mathrm{U}$ Mon, and R Sge, which are the only objects of our northern sample that were not detected. Wide spectral bands are shown, centered on the stellar velocity expected from optical observations. See a summary of the observational parameters in Sect. 2 and Table 3.

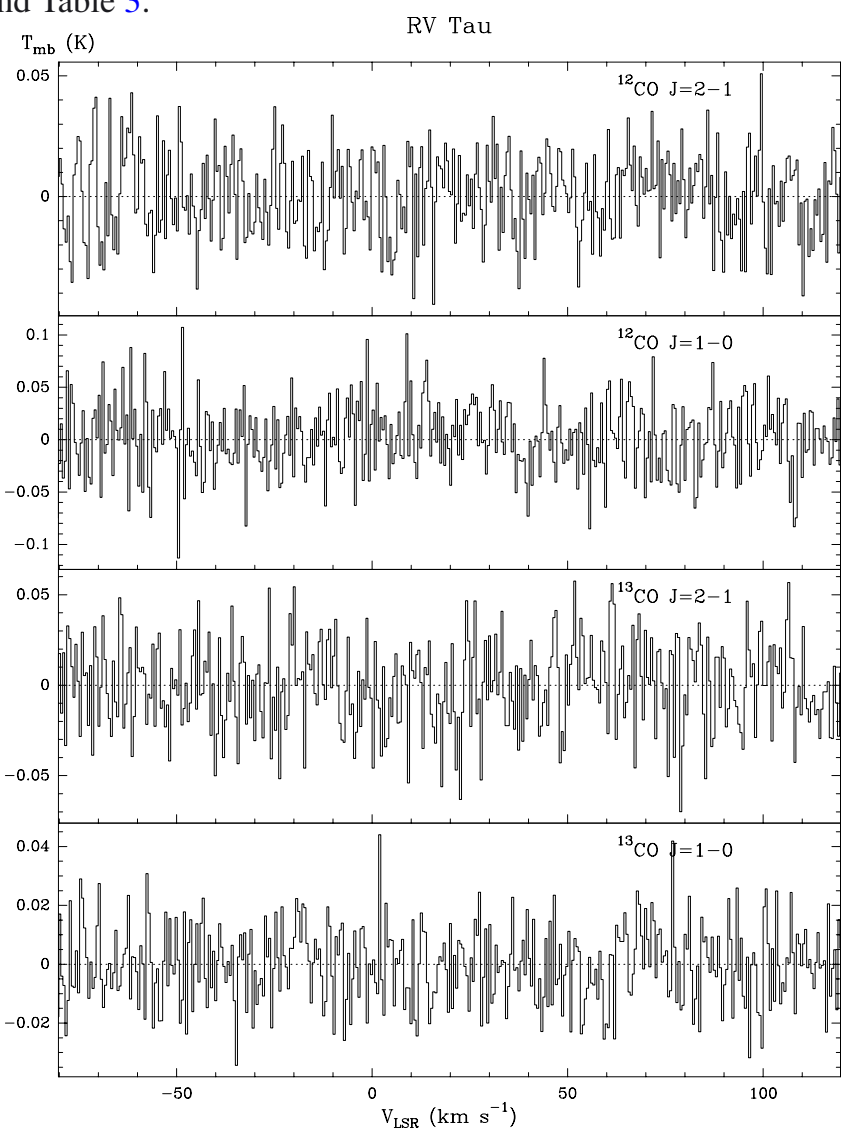

Fig. A.1. $30 \mathrm{~m}$ telescope observations of RV Tau. No line is detected.

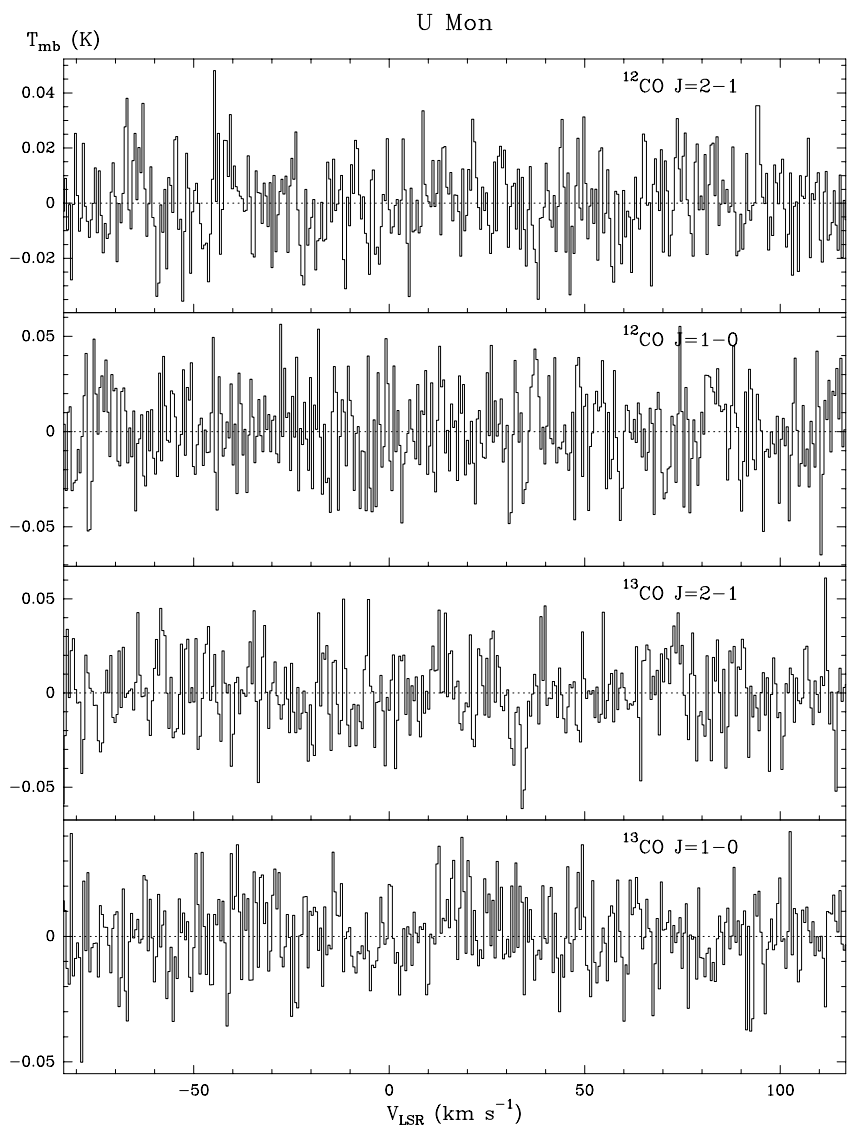

Fig. A.2. $30 \mathrm{~m}$ telescope observations of $\mathrm{U}$ Mon. No line is detected.

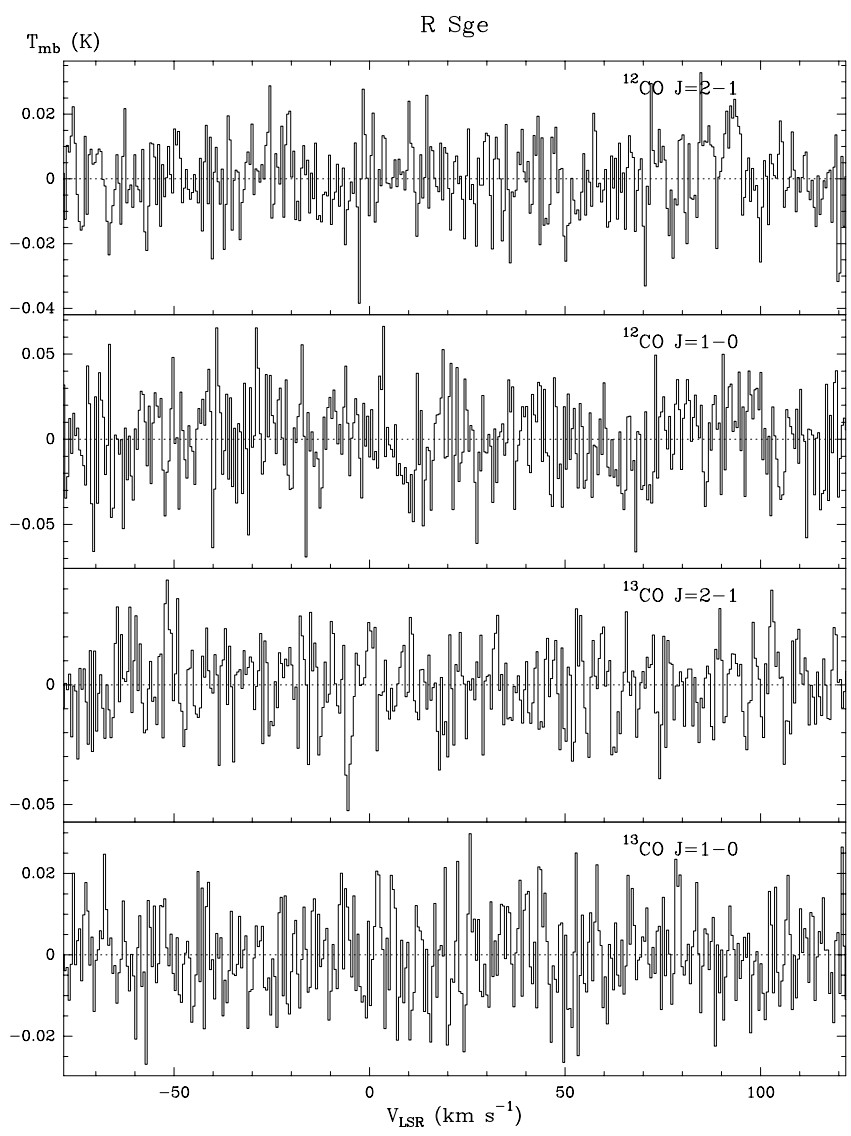

Fig. A.3. $30 \mathrm{~m}$ telescope observations of R Sge. No line is detected. 


\section{Appendix B: APEX observations of southern sources: spectra and disk mass estimates}

We have also observed southern post-AGB stars using APEX in the ${ }^{12} \mathrm{CO} J=3-2$ and $J=2-1$ transitions, the source sample is given in Table 2 (we give the same parameters as in Table 1 for the northern sources). Because of the lack of ${ }^{13} \mathrm{CO}$ observations and the poorer quality of the data, which include spectra dominated by interstellar (IS) contamination, these results are not analyzed with the same detail as those of northern sources. When strong IS contamination is present, it is even impossible to give a reasonable upper limit to the post-AGB source emission, since the IS contribution can be very intense, both positive and negative, and practically impossible to predict. The observational procedure is described in Sect. 3.2, and a summary of the line parameters is given in Table 4 (similar to that given in Table 3), and the observed profiles are given in Figs. B.1 to B.10. To derive mass values for the disk in the detected sources, we first compared the mass values derived from the ${ }^{13} \mathrm{CO}$ $J=1-0$ integrated main-beam temperatures (our best estimates, in Table 5) with those derived from ${ }^{12} \mathrm{CO} J=2-1$ (very probably underestimates), in both cases following the method described in Sect. 5. We have not considered the mass derived for R Sct and IRAS 20056+1834, in which the contribution of an additional component, probably in expansion, is largely dominant. The result is shown in Fig. B.11, the mass values derived from ${ }^{12} \mathrm{CO} J=2-1$ are approximately ten times lower than those obtained from ${ }^{13} \mathrm{CO} J=1-0$, which are the best estimates. We will accordingly estimate the mass of the southern sources from their ${ }^{12} \mathrm{CO} J=2-1$ intensities (also considering SEST observations of IRAS 08544-4431 by Maas et al. 2003) and correct them by a factor 10. In the calculation of the disk mass values from the SEST and APEX data, we consider that the APEX beam solid angle (at $230 \mathrm{GHz}$ ) is about 4.7 times greater than that of the $30 \mathrm{~m}$ telescope and that the beam of the SEST is about 4 times greater. The (uncertain) mass estimates derived in this way for our four detected southern sources are given in Table 5 (last four lines).

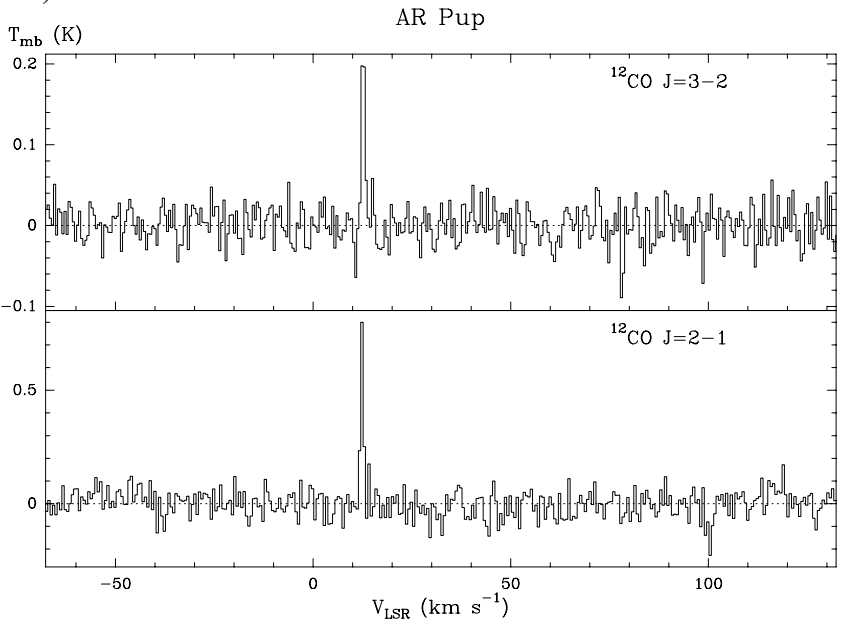

Fig. B.1. APEX observations of AR Pup. No circumstellar line is detected; only a narrow interstellar feature is found.

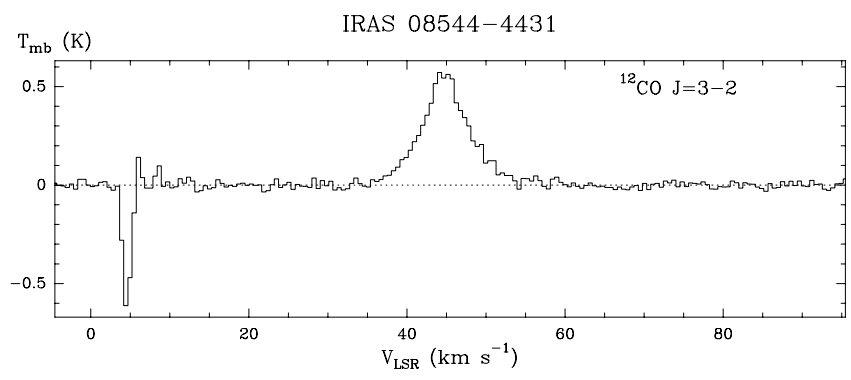

Fig. B.2. APEX observations of IRAS 08544-4431. ${ }^{12} \mathrm{CO} J=3-2$ is detected (see previous data by Maas et al. 2003).

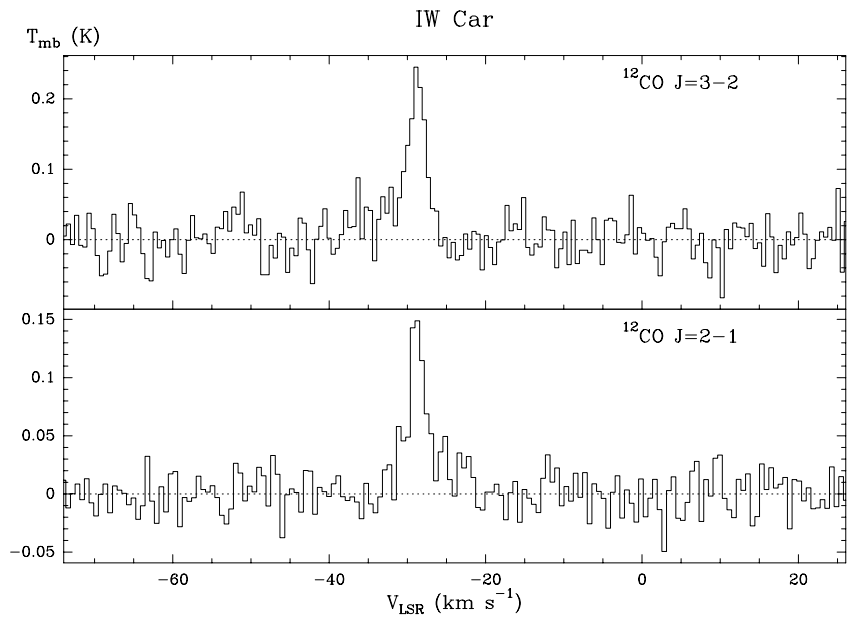

Fig. B.3. APEX observations of IW Car. Both lines are detected.

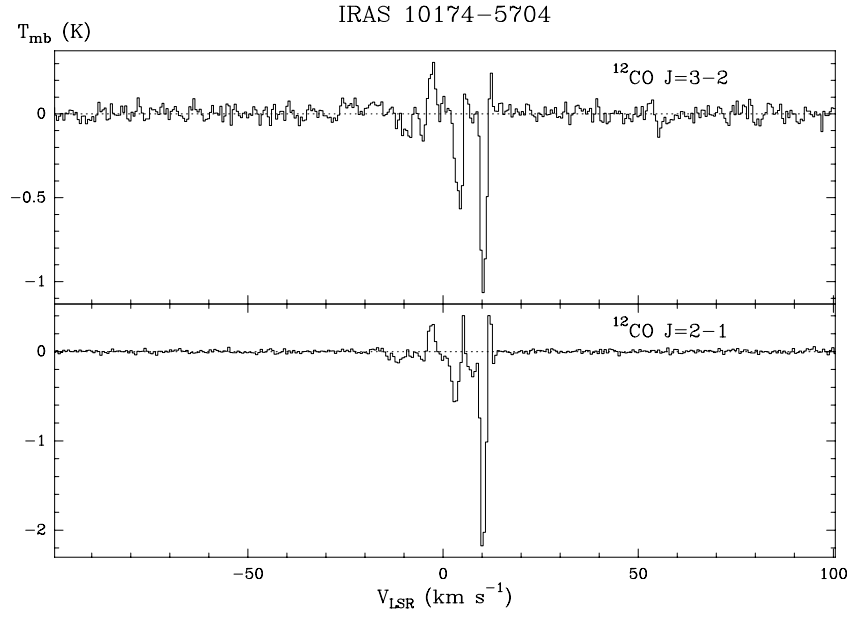

Fig. B.4. APEX observations of IRAS 10174-5704. Strong interstellar contamination at the relevant velocities prevents any conclusion on the emission from our source. 
V. Bujarrabal et al.: Extended rotating disks around post-AGB stars

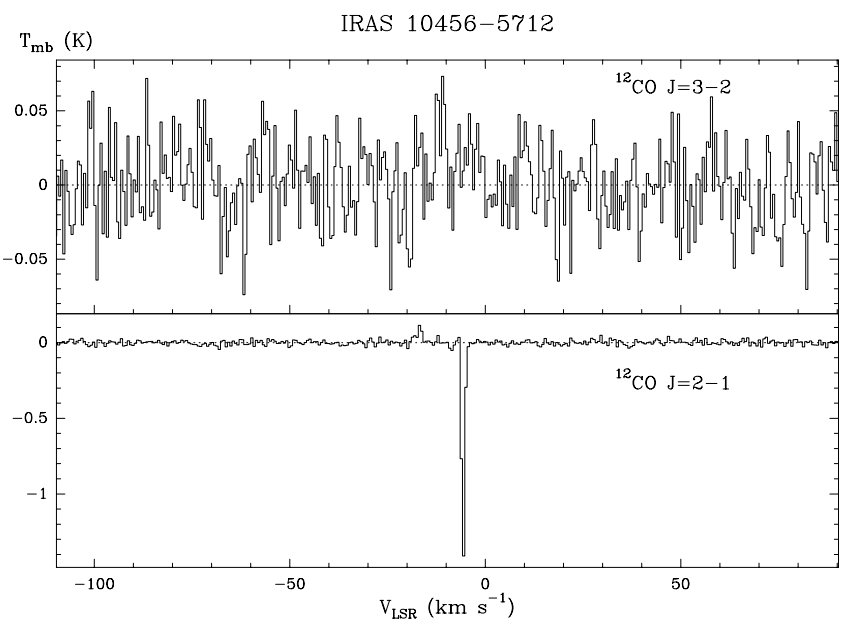

Fig. B.5. APEX observations of IRAS 10456-5712. Strong interstellar contamination at the relevant velocities prevents any conclusion on the emission from our source.

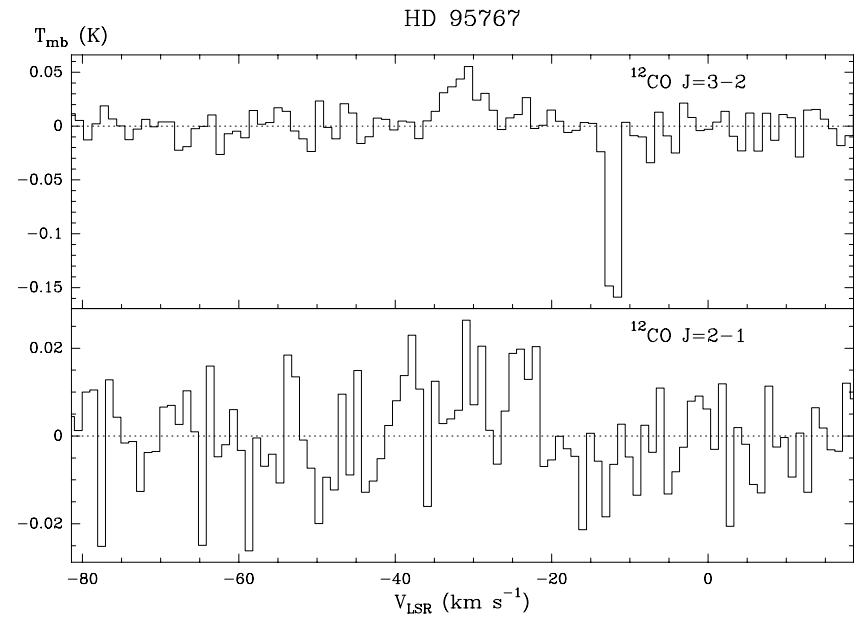

Fig. B.6. APEX observations of HD 95767. Emission is detected in $J=3-2$, at the velocity found from optical spectroscopy, and tentatively in $J=2-1$.

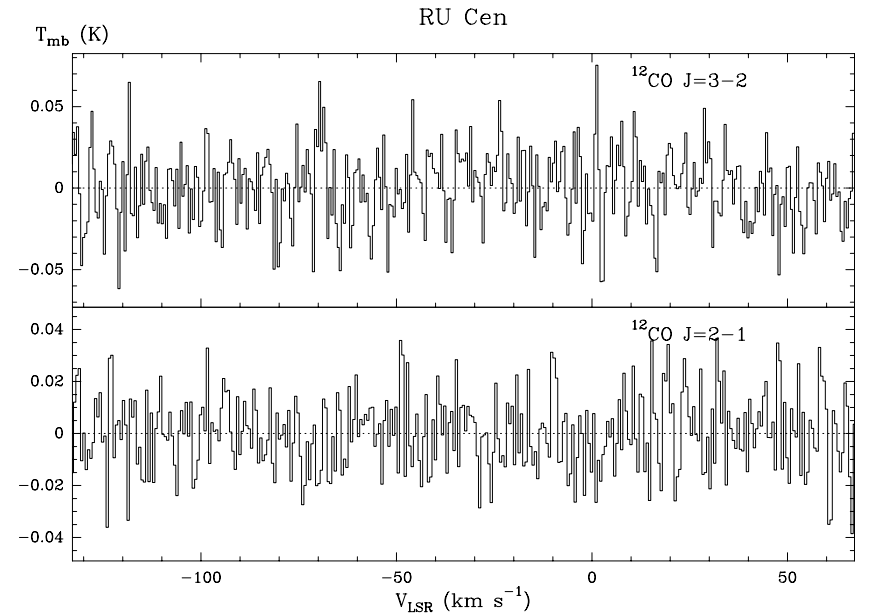

Fig. B.7. APEX observations of RU Cen. No circumstellar line is detected.

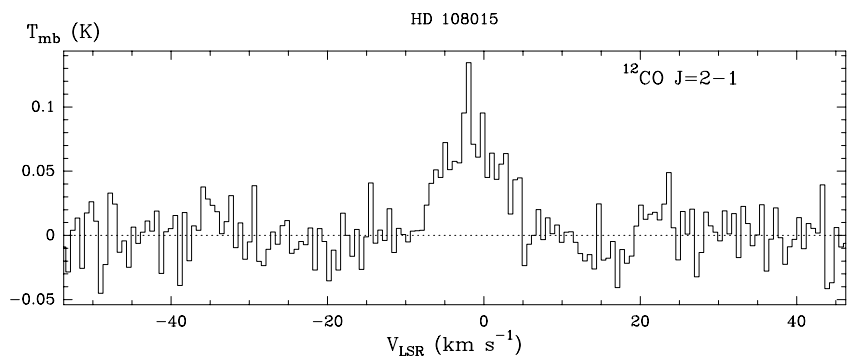

Fig. B.8. APEX observations of HD 108015. ${ }^{12} \mathrm{CO} J=2-1$ is detected.

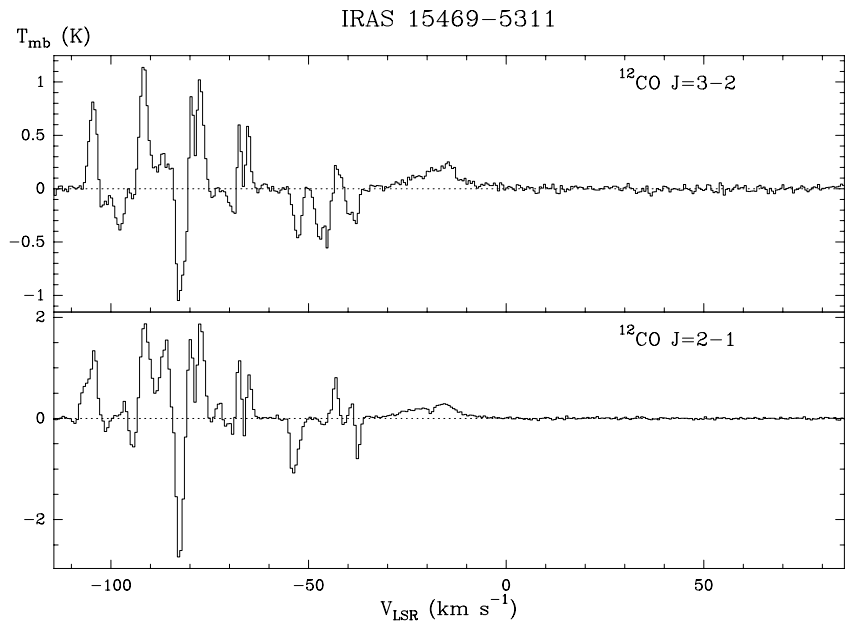

Fig. B.9. APEX observations of IRAS 15469-5311. Strong interstellar contamination at the relevant velocities prevents any conclusion on the emission from our source.

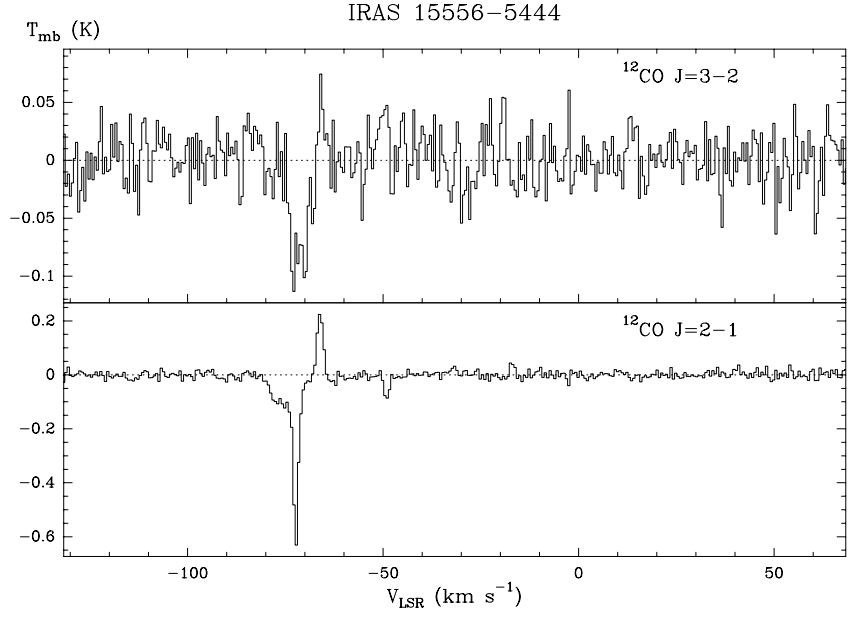

Fig. B.10. APEX observations of IRAS 15556-5444. Strong interstellar contamination at the relevant velocities prevents any conclusion on the emission from our source. 
A\&A 557, A104 (2013)

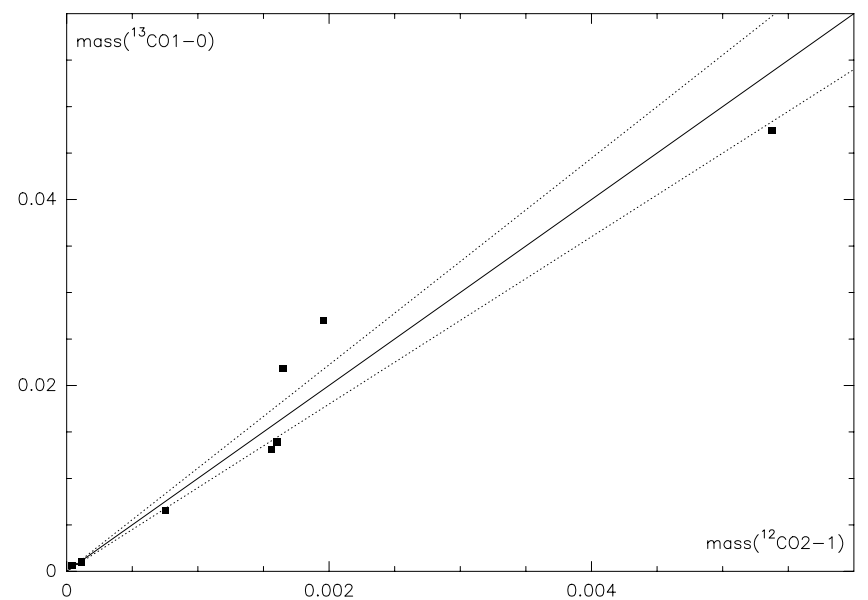

Fig. B.11. Relation between the mass values $\left(M_{\odot}\right)$ derived respectively from ${ }^{13} \mathrm{CO} J=1-0$ and ${ }^{12} \mathrm{CO} J=2-130 \mathrm{~m}$ telescope data for the objects in which both determinations can be performed (Sect. 5). Results from ${ }^{13} \mathrm{CO} J=1-0$ are expected to be the most accurate ones and those from ${ }^{12} \mathrm{CO} J=2-1$ are underestimates. The continuous line represents a factor of ten between both estimates, and the other lines represent deviations of a $10 \%$ from this ratio. 